\title{
IMPROVED CRACK TIP ENRICHMENT FUNCTIONS AND INTEGRATION FOR CRACK MODELING USING THE EXTENDED FINITE ELEMENT METHOD
}

\author{
Nicolas Chevaugeon, Nicolas Moës \& Hans Minnebo
}

LUNAM Universite, GeM UMR6183, Ecole Centrale de Nantes, 1 Rue de la Noe, 44321, Nantes, France

*Address all correspondence to Nicolas Chevaugeon, E-mail: nicolas.chevaugeon@ec-nantes.fr

\begin{abstract}
This paper focuses on two improvements of the extended finite element method (X-FEM) in the context of linear fracture mechanics. Both improve the accuracy and the robustness of the X-FEM. In a first contribution, a new enrichment strategy is proposed to take into account the singular stress field at the crack tip that is meant to replace the traditional four-crack-tip enrichment functions. The efficiency of the new approach is demonstrated on mesh convergence experi-ments for twodimensional straight and curved crack problems, using first- and second-order shape functions, both in terms of convergence rates and in terms of condition number of the system to solve. The second contribution revisits the problem of the numerical integration of the stiffness operator when singular functions like the tip enrichment functions are used. An original algorithm to build accurate and fast integration rules for elements in the enrichment zone, touch-ing the crack tip singularity, or not, is presented. The effects on convergence rate of the choice of the integration rule are illustrated on numerical examples.
\end{abstract}

KEY WORDS: X-FEM, cracks, singular function integration, LEFM

\section{INTRODUCTION}

The paper presents some improvements to the extended finite element method (X-FEM) in the context of linear fracture mechanics. The goal is to provide better convergence rate and robustness to the method. Most FEM industrial codes provide up to a second-order finite element, with a corresponding optimal second-order $h$ convergence rate of the energy norm error when applied to regular problems. The X-FEM, beyond relaxing the constraint on the mesh, also has the potential to reach optimal convergence rate in the presence of a stress singularity such as a crack. It is generally considered achieved when first-order shape functions are used (Béchet et al., 2005; Laborde et al., 2005). Using a geometrical enrichment strategy and a sufficiently accurate integration rule, a first-order convergence rate has already been demonstrated. Moving to higher order while keeping the optimal convergence rate is much more difficult. This is our ultimate goal and the contributions of this paper are steps toward that goal.

The outline of the paper is as follows: In the first section, the X-FEM modeling strategy for cracks is briefly recalled, with references and comparisons to the variants introduced by the generalized finite element method (GFEM). Special care is focused toward the definition of the enrichment zone, either topological or geometrical. In this first section, the vectorial crack tip enrichment strategy is be presented, along with some details on its implementation. The second section is devoted to the analysis and the construction of a new quadrature rule meant to reduce the integration error in the stiffness matrix, due to the presence of singular terms coming from the tip enrichment functions. In the last section, the proposed improvements are tested on some benchmarks where high-order accuracy is achieved. Optimal order of convergence is obtained, for both first- and second-order shape functions, with a reasonable condition number for the stiffness matrix, compared to the previous method. Lastly, taking advantage of the structure of the vector enrichment functions, a very fast stress intensity factor extractor is presented. Its usefulness as compared to G-theta domain integral methods is discussed. Finally, the paper ends with some conclusions. 


\section{THE X-FEM MODELING OF CRACKS}

\subsection{Problem Statement}

We place ourselves within the context of linear elasticity. On a discretized domain $\Omega^{h}$, where $h$ is a discretization parameter (element size) bounded by $\Gamma^{h}$, the weak form of the discretized equilibrium equations may be written as follows:

Find $\mathbf{u}^{h}$, the discretized displacement field $\in \mathbf{V}^{h}$, such that

$$
\int_{\Omega^{h}} \mathbf{B}\left(\mathbf{u}^{h}, \mathbf{v}^{h}\right) d \Omega=\int_{\Gamma_{t}} \mathbf{L}\left(\mathbf{v}^{h}\right) d \Gamma \forall \mathbf{v}^{h} \in \mathbf{V}_{0}^{h}
$$

where $\mathbf{V}^{h}$ is the trial space for the displacement field, over the mesh that discretizes the domain $\Omega$, that fulfills the Dirichlet boundary condition on $\Gamma_{u}^{h}$, and $\mathbf{V}_{0}^{h}$ is the corresponding space with a homogeneous Dirichlet boundary condition. $\mathbf{B}$ is a bilinear operator, defined as $\mathbf{B}(\mathbf{u}, \mathbf{v})=\nabla^{s} \mathbf{u}^{h}: \mathbf{D}: \nabla^{s} \mathbf{v}^{h}$, where $\nabla^{S} \mathbf{u}$ is the symmetric part of the gradient tensor of $\mathbf{u}$ (e.g., the linearized strain tensor), and $\mathbf{D}$ is the fourth-order elasticity tensor. $\mathbf{L}$ is a linear operator corresponding to the Neumann boundary condition on $\Gamma_{t}: \mathbf{L}(\mathbf{u})=\mathbf{t} . u$, where $\mathbf{t}$ represents the prescribed tractions known on $\Gamma_{t}$.

The domain $\Omega$ is then cut by a traction-free crack. In the context of X-FEM, the mesh does not conform to the crack and alternative representations for the geometry of the crack need to be used.

\subsection{Geometric representation of the crack}

The modeling of three-dimensional (3D) cracks not aligned with the mesh using the partition of unity was first developed in Sukumar et al. (2000). The description of the crack location was explicit in this paper.

Later, in Moës et al. (2002), a more flexible level set representation was introduced, the representation used in the present work, which we describe in the following. The crack location is given by two level sets. The normal level set, $l_{\mathrm{n}}$, corresponds to the signed distance to the crack surface. The tangential level set $l_{\mathrm{t}}$ corresponds the signed distance to the front (or to be more precise, the distance to a surface passing through the front and orthogonal to the crack surface). The crack front is given by the set of points were both $l s_{n}$ and $l s_{t}$ are zero, whereas the crack is given by the set of points for which $l s_{n}=0$ and $l s_{t} \leq 0$. The iso-zero of the two level sets are depicted in Fig. 1 close to a crack front portion.

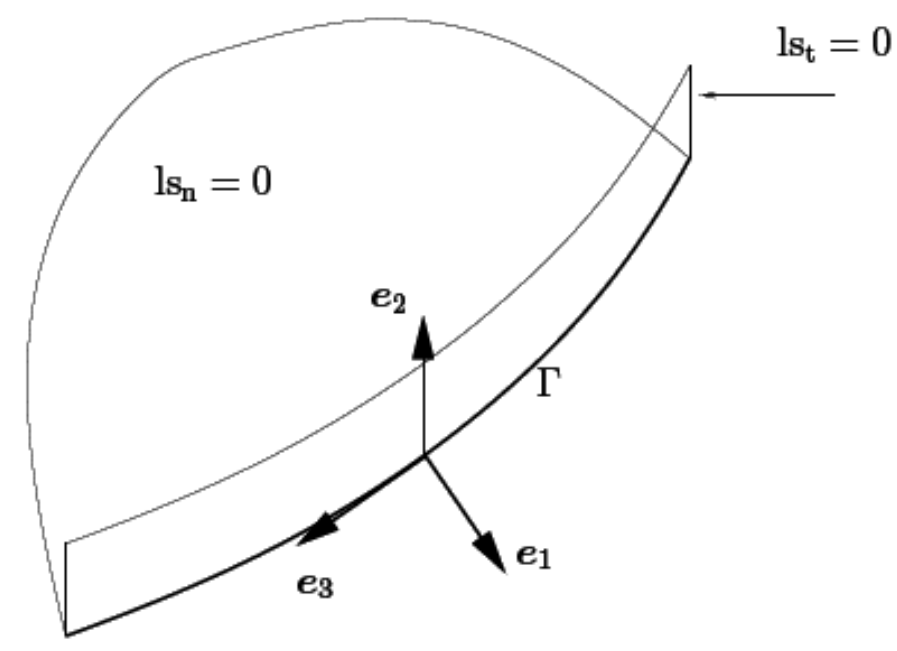

FIG. 1: Level set representation of a crack and definition of the local axis along the crack front. 
Numerically, the level sets are discretized as a linear finite element approximation, over the mesh that is used for the computation of the displacement field. It is, of course, possible to use another mesh to represent the level set. For example, it was proposed to represent the level set on a finer mesh, or on a structured or octree type of mesh (Legrain et al., 2012; Prabel et al., 2007; Sukumar et al., 2008). The latter versions are sometimes more memory efficient, and more efficient propagation algorithms can be used to update the position of the crack when performing crack propagation analysis. Using an implicit representation of the crack such as the one presented, has some drawbacks. Indeed, the crack surface, if simple enough, could be described by a parametric surface, or by a mesh. The memory cost of such a representation would be in most cases much smaller than the cost of representing two discrete level sets over a whole mesh. Those claims could nonetheless be more balanced, since the level sets need only to be defined in a narrow band containing the crack surface (Osher and Fedkiw, 2002; Sethian, 1999).

In the context of crack propagation, the crack front represented by the level set $l_{s_{t}}$ is able to take any topology. Updating a mesh of the crack front, on the other hand, can be more difficult.

\subsection{Crack Tip Enrichment: Classical Version}

The commonly used X-FEM approximation field is as follows. If no crack is present, the displacement field $\mathbf{u}(\mathbf{x})$ over the body located in $\Omega$ is approximated by

$$
\mathbf{u}^{h}(\mathbf{x})=\sum_{i \in[1: \operatorname{dim}]} \sum_{I \in \mathcal{N}} N_{I}(\mathbf{x}) u_{I i} \mathbf{E}_{i}
$$

where $\mathcal{N}$ is the set of all nodes in the mesh, $N_{I}$ are the classical $C^{0}$ shape functions, and $u_{I i}$ are the displacement degrees of freedom attached to node $I$ in direction $\mathbf{E}_{i}$, where the $\mathbf{E}_{i}$ form a global orthonormal basis, and $\operatorname{dim}$ is the number of spacial dimensions of the problem. Introducing a crack in the mesh yields the following enriched approximation:

$$
\begin{aligned}
\mathbf{u}^{h}(\mathbf{x}) & =\sum_{i \in[1: d i m]} \sum_{I \in \mathcal{N}} N_{I}(\mathbf{x}) u_{I i} \mathbf{E}_{i}+\sum_{i \in[1: d i m]} \sum_{I \in \mathcal{N}_{\text {crack }}} N_{I}(\mathbf{x}) H\left[\mathrm{ls}_{\mathrm{n}}(\mathbf{x})\right] h_{I i} \mathbf{E}_{i} \\
& +\sum_{i \in[1: d i m]} \sum_{I \in \mathcal{N}_{\text {tip }}} \sum_{\alpha} N_{I}(\mathbf{x}) F_{\alpha}\left[\mathrm{s}_{\mathrm{n}}(\mathbf{x}), \mathrm{ls}_{\mathrm{t}}(\mathbf{x})\right] a_{I i \alpha} \mathbf{E}_{i}
\end{aligned}
$$

in which

- $\mathcal{N}_{\text {crack }}$ is the set of nodes whose support (union of the elements connected to the node) is completely cut into two by the crack. These nodes are enriched by the generalized Heaviside function:

$$
H\left[\mathrm{l}_{\mathrm{n}}(\mathbf{x})\right]=\operatorname{sign}\left[\operatorname{ls}_{\mathrm{n}}(\mathbf{x})\right]
$$

$\operatorname{sign}(x)=-1$ if $x<0, \operatorname{sign}(x)=+1$ if $x \geq=0$.

$-\mathcal{N}_{\text {tip }}$ is the set of nodes enriched by the tip enrichment functions:

$$
\left[F_{\alpha}\right]=\left[\sqrt{r} \sin \frac{\theta}{2}, \sqrt{r} \cos \frac{\theta}{2}, \sqrt{r} \sin \frac{\theta}{2} \sin \theta, \sqrt{r} \cos \frac{\theta}{2} \sin \theta\right]
$$

where

$$
r=\sqrt{\mathrm{ls}_{\mathrm{n}}^{2}+\mathrm{ls}_{\mathrm{t}}^{2}}, \quad \theta=\tan ^{-1}\left(\frac{\mathrm{ls}_{\mathrm{n}}}{\mathrm{ls}_{\mathrm{t}}}\right)
$$

- $h_{I i}$ are the degrees of freedom associated to the Heaviside enrichment function attached to node $I$, in direction $i$.

- $a_{I i \alpha}$ are the degrees of freedom associated to the $\alpha$ crack tip enrichment function attached to node $I$, in direction $i$. 
The set $\mathcal{N}_{\text {tip }}$ must at least contain the nodes whose supports touch the crack front. Similarly to what was introduced in Béchet et al. (2005) and Laborde et al. (2005), we can distinguish topological and geometrical enrichment. We denote by $V^{\text {geo }}(R)$ the set of elements for which at least one node is at a distance $r$ to the crack front smaller or equal to $R$. The distance $r$ is computed by Eq. (1.6). We denote by $V^{\text {topo }}(n)$ the set of elements composing $n$ layers of elements around the front. The precise definition of the layers follows. The first layer contains all the elements touching the front. The second layer is defined as the elements connected to the nodes of the elements of the first layer. The third layer is defined as the elements connected to the nodes of the elements of the second layer and so on. For all the numerical examples presented in the presented here, we use the geometrical enrichment strategy where $\mathcal{N}_{\text {tip }}$ is the set of nodes of $V^{\text {geo }}(R)$. It was shown, for example, in Béchet et al. (2005) that it was required to obtain an optimal rate of convergence in energy error norm. For the rest of the paper, we refer to the use of the four $F_{\alpha}$ crack tip enrichment functions as the scalar enrichment or scalar tip enrichment to differentiate with the new enrichment that is presented in the next section.

\subsection{Crack Tip Enrichment: Updated Version}

\subsubsection{Presentation}

In the present paper we propose to investigate an updated version of the X-FEM enrichment we just discussed. The main difference is that instead of using the four $F_{\alpha}$ scalar enrichment functions, we propose to use three $\mathbf{K}_{\alpha}$ vector enrichment functions. Equation (1.3) then reads

$$
\begin{aligned}
\mathbf{u}(\mathbf{x}) & =\sum_{i \in[1: d i m]} \sum_{I \in \mathcal{N}} N_{I}(\mathbf{x}) u_{I i} \mathbf{E}_{i}+\sum_{i \in[1: d i m]} \sum_{I \in \mathcal{N}_{\text {crack }}} N_{I}(\mathbf{x}) H\left[\mathrm{ls}_{\mathrm{n}}(\mathbf{x})\right] h_{I i} \mathbf{E}_{i} \\
& +\sum_{I \in \mathcal{N}_{\text {tip }}} \sum_{\alpha} N_{I}(\mathbf{x}) \mathbf{K}_{\alpha}\left[\mathrm{ls}_{\mathrm{n}}(\mathbf{x}), \mathrm{ls}_{\mathrm{t}}(\mathbf{x})\right] a_{I \alpha}
\end{aligned}
$$

In this updated version, only the last sum differs slightly. The $a_{I \alpha}$ are now scalar degrees of freedom and the vectorial nature of the displacement field is embedded in the enrichment functions. We make the choice to use three vector enrichment functions that are a dimensionless version of the three Irwin asymptotic opening modes (Irwin, 1957).

$$
\begin{gathered}
\mathbf{K}_{1}=\sqrt{r} \cos \frac{\theta}{2}(\kappa-\cos \theta)\left[\mathbf{e}_{1}(\mathbf{x})+\mathbf{e}_{2}(\mathbf{x})\right] \\
\mathbf{K}_{2}=\sqrt{r} \sin \frac{\theta}{2}(\kappa+2+\cos \theta) \mathbf{e}_{1}(\mathbf{x})+\sqrt{r} \cos \frac{\theta}{2}(\kappa-2 \cos \theta) \mathbf{e}_{2}(\mathbf{x}) \\
\mathbf{K}_{3}=\sqrt{r} \sin \frac{\theta}{2} \mathbf{e}_{3}(\mathbf{x})
\end{gathered}
$$

where $k=3-4 v$, with $v$ the Poisson ratio, in 3D or the plane strain case.

\subsubsection{Bibliographic Discussion}

While the idea of using these enrichment functions seemed original to us at the time of development, further review of the bibliography showed that the idea was already used in a similar form in the G-FEM context. While G-FEM and X-FEM methods appeared approximately at the same time and were both applications of the partition-of-unity method and were both applied to the modelization of cracks, they were initially quite different. The two methods evolved since then borrowing ideas from each other to the point that it is difficult to distinguish them now (Belytschko and Fries, 2010). If we refer to early applications for cracks $(1999,2000)$ the introduction of discontinuity inside an element was done using a discontinuous partition of unity in the G-FEM, while in the case of the X-FEM, the discontinuity was introduced via Heaviside enrichment multiplied by a linear, continuous partition of unity. In the original G-FEM, the displacement space is defined as

$$
u=\sum q_{i} \phi_{i}(x) N_{i}
$$


where the $\phi_{i}(x)$ are constructed from Shepard function, using the visibility criteria, and the $N_{i}$ are the classical finite element shape functions. In the original X-FEM the displacement space is defined as

$$
u=\sum q_{i} N_{i}+\sum h_{i} N_{i} H\left[\mathrm{ls}_{\mathrm{n}}(x)\right]
$$

where the $N_{i}$ are the classical finite element shape functions and $H$ is the Heaviside function, defined on top of the normal level set that gives the distance to the crack surface. More recent papers in G-FEM seem to have adopted a formulation closer to X-FEM, where the discontinuity is now introduced via the enrichment. Enriching the elements touching the crack tip directly with vectorial functions that are the asymptotic expansion of the exact solution appeared, to our knowledge, in G-FEM in Duarte et al. (2000), where it was applied to describe corners or wedges and, at the limit when the angle goes to 0 , cracks. The discrete displacement field is enriched around a corner using vectorial functions that are directly the asymptotic expansion of the exact solution. In this paper, the mesh was conformed to the corner. In the following paper (Duarte et al., 2001), the method is explicitly applied to cracks and the "WA" criteria is used to choose a node enriched by the asymptotic expansion. WA criteria refer to "wrap around nodes," e.g., all nodes belonging to elements that intersect the crack front. At this time, the enrichment is therefore only "topologic" in our designation. The enrichment proposed in this paper is indeed the same as the one proposed in Duarte et al. (2000), but at the time of this paper, the need to have a geometric criteria instead of a topological in order to get optimal convergence order was not yet realized, and the idea of using a level set to represent the crack was not used. In a more recent paper (Pereira et al., 2009), the same group proposed another enrichment strategy, inspired by the initial one, where the three vectorial asymptotic displacement field functions are split in six enrichment functions according to the tangential and normal directions. This approach permits the precise description of the direction of the crack to be avoided, while still limiting the number of degrees of freedom per enriched node to 6 (to be compared to the 12 enrichment functions with scalar enrichment). In this paper, the author used topologic enrichment, claiming that geometrical enrichment only works for planar crack. As shown in the examples Section 3, the geometric enrichment works perfectly in our case, even for nonplanar cracks. Until now, all papers that model crack using the X-FEM and crack tip enrichment used the basis proposed initially, i.e., the four scalar enrichment functions 1.5 , applied separately in each space direction. In view of the bibliography, the originality of the present paper is therefore not to use the presented vector enrichment functions, but rather to use them in the context of geometrical enrichment, based upon a level set representation of the crack and realizing the consequences on the conditioning of the stiffness matrix, while showing an optimal rate of convergence for both straight and curved cracks.

\subsubsection{Implementation Issues}

The three vector enrichment functions (1.8-1.10) are defined as a function of the local crack basis, that, in general depends on $\mathrm{x}$. Therefore, to ensure continuity of the displacement field, at first sight, it seems that the local basis has to be defined continuously over the enrichment zone. It would mean that one needs to build from the gradient of the discretized level set, a continuous field of moving local basis. Furthermore, when computing the gradient of the proposed enriched function, a curvature term $\left(\partial \mathbf{e}_{i}\right) / x_{j}$ would appear. Even if this term might be neglected when the curvature of the crack is small compared to the element size in the enrichment zone, it seems a difficult task to implement a robust version using this point of view. In fact, we propose a much easier alternative. We propose to define a local crack basis discretization per enriched node. This local basis needs only to be defined over the support of the enriched node. Since the enrichment function associated to an enriched node is multiplied by the finite element shape functions associated to the node, the resulting function is always zero on the boundary and outside of the support. This alleviates the continuity constrain over the local crack basis discretization: for each enriched node, its associated local crack basis discretization needs only to be continuous over its support, resulting in an overall continuous field. To simplify the discretization even more, we choose a constant approximation of each local crack basis per enriched node over the node's support. This permits the computation of the curvature term to be avoided. Figure 2 illustrates a typical situation. On this small mesh, suppose that only nodes A and B are enriched. When computing the stiffness matrix on the gray element, the contribution of the enrichment of node A and B must be taken into account. When computing the enrichment attached to node A, we use the local basis defined for the support of node A, and respectively, when 


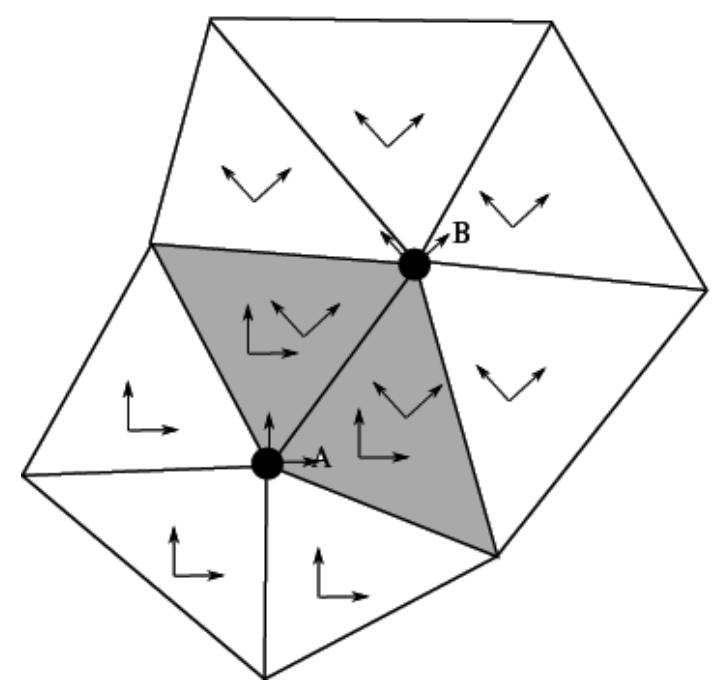

FIG. 2: The support of two nodes (large dot), with two local basis, one belonging to each node.

computing the enrichment attached to node $\mathrm{B}$, we use the local basis defined for the support of node $\mathrm{B}$. The number of local basis defined over an element is equal to the number of supports of enriched nodes this element is a part of. The enrichment function is now a function of the enriched node. This is reflected in the following rewriting of the last term of Eq. (1.7):

$$
\sum_{I \in \mathcal{N}_{\mathrm{tip}}} \sum_{\alpha} N_{I}(\mathbf{x}) \mathbf{K}_{\alpha}^{I}\left[\mathrm{ls}_{\mathrm{n}}(\mathbf{x}), \mathrm{ls}_{\mathrm{t}}(\mathbf{x})\right] a_{I \alpha}
$$

where the dependence of the enrichment function on the node it is attached to appears clearly, and where

$$
\begin{gathered}
\mathbf{K}_{1}^{I}=\sqrt{r} \cos \frac{\theta}{2}(\kappa-\cos \theta)\left(\mathbf{e}_{1}^{I}+\mathbf{e}_{2}^{I}\right) \\
\mathbf{K}_{2}^{I}=\sqrt{r} \sin \frac{\theta}{2}(\kappa+2+\cos \theta) \mathbf{e}_{1}^{I}+\sqrt{r} \cos \frac{\theta}{2}(\kappa-2 \cos \theta) \mathbf{e}_{2}^{I} \\
\mathbf{K}_{3}^{I}=\sqrt{r} \sin \frac{\theta}{2} \mathbf{e}_{3}^{I}
\end{gathered}
$$

where $\mathbf{e}_{i}^{I}$ is the discretized value of the $i$ th basis vector of the moving local axis, at node $I$. In our implementation of the X-FEM, the two level sets are discretized with linear shape function over the mesh. We can therefore compute their gradient, constant in each element, and from them construct a constant orthogonal basis per element that approximates the local crack tip axis. But we need a constant value per support. To obtain this value, we simply take the weighted average of the constant gradients over each element in the support of the enriched node, and from these values, construct an orthogonal basis. This scheme is very efficient and easy to implement.

This concludes our first part. We reviewed the X-FEM for linear fracture mechanics, covering the representation of the geometry and proposing a different crack tip enrichment strategy. Our vector enrichment strategy is tested and discussed more in part 3, but first we must cover the problem of the integration of the elasticity operator (the stiffness matrix) over enriched elements.

\section{INTEGRATION RULE}

Integration of the bilinear form $B\left(\mathbf{v}^{h}, \mathbf{u}^{h}\right)$, for each enriched element, is known to be problematic. Indeed, leading terms of the expression to integrate include terms such as $1 / r$ due to the tip enrichment, and the functions to integrate 
are discontinuous across the crack surface. In the context of two- or three-dimensional problems, there is no hope for an exact integration of the bilinear form over a tip-enriched element. Numerical integration is, of course, needed. From the start of the X-FEM, some papers already tried to deal with this issue: how to obtain an efficient integration scheme so that the error in the integration of the bilinear form doesn't pollute the results so much as it degrades the convergence properties. Recent references on the subject are, among others, Béchet et al. (2005), Park et al. (2009), and Mousavi and Sukuvar (2010), which used ideas developed outside of the X-FEM context in Duffy (1982) and Nagarajan and Mukherjee (1993). The first step was to cut the element to integrate into a series of cells along the crack surface and then use classic Gauss-like integration rules over each cell, usually of higher order than the rule needed to integrate classic terms. This permits us to have exact integrations of terms that come from classic shape function and Heaviside enrichment, but leaves in most cases, many errors for terms that involve the tip enrichment functions. The situation was improved when special integration rules where used for cells that have one node on the crack tip. We think that this approach is not always sufficient. In the present paper, we propose and implement, starting from a singular mapping for singular elements, a new strategy that permits all the enriched elements to be integrated with high precision, not only the ones that touch the crack tip, for the two-dimensional case.

\subsection{Fundamental Background - Integration of Weakly Singular Functions Over a Segment}

In the present section we analyze a very simple one-dimensional case in order to explain the problems we want to cope with in the more general case. Integration of a function over an interval using a Gauss-Legendre rule is known to converge exponentially to the exact solution if the function to integrate is regular $\left(C^{\infty}\right)$. In our case, we are interested in the computation of functions with a singularity, or a singularity in one of their gradients, at the position of the crack tip. To start the analysis, let us consider the quadrature of $\sqrt{(|r|)}$ over the interval $[-1: 1]$, using Gauss-Legendre points. Since the function has indefinite derivative at zero, the error in the integral does not converge exponentially. A first improvement would be to cut the interval at point $r=0$ and perform a numeric integral over $[-1: 0[$ and then ] $0: 1]$. As can be seen in Fig. 3, in the first case, the error oscillates while slowly converging to zero with the number of points: in the second case, the convergence is still slow, but does not oscillate anymore. This first basic observation will motivate the choice of cutting the elements into cells that can contain the singularity only on their corner, not inside. Let us now pursue the analysis by considering the quadrature of $\sqrt{r}$ over the interval $[\epsilon: 1+\epsilon]$ with $\epsilon \geq 0$. On the left side of Fig. 4, we report the relative error of the quadrature of $\sqrt{x}$ as a function of the number of Gauss-

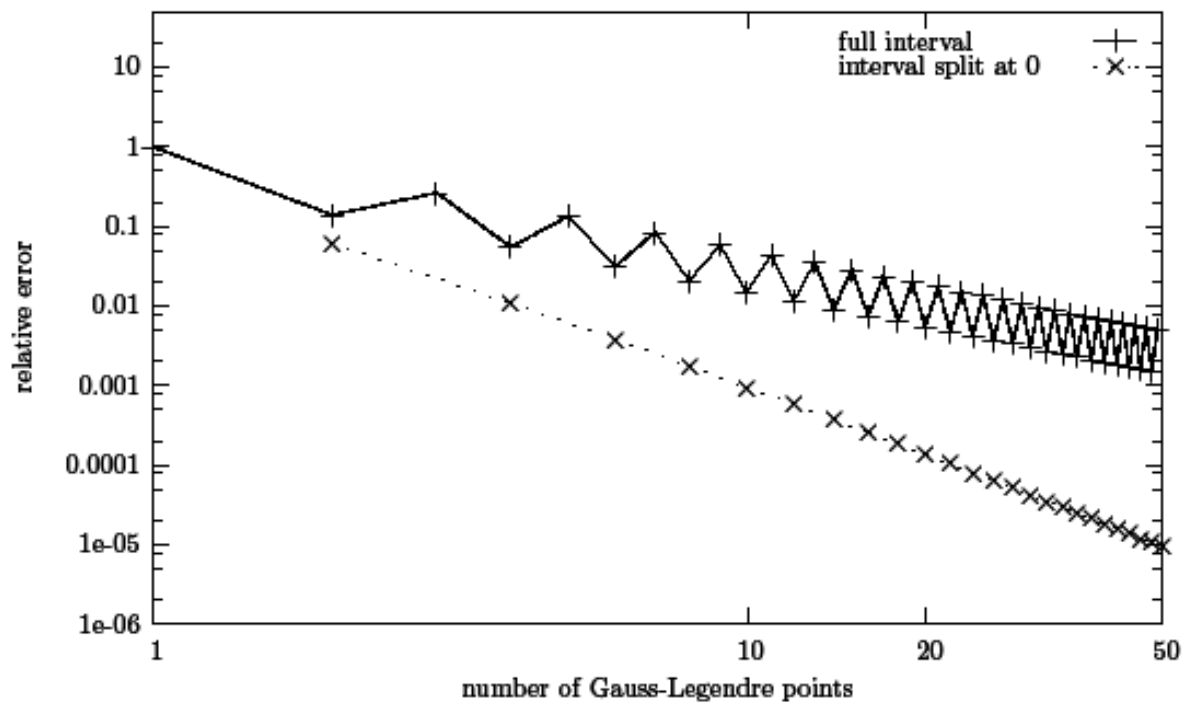

FIG. 3: Integration error of $\sqrt{(\|r\|)}$ over $[-1: 1]$. 

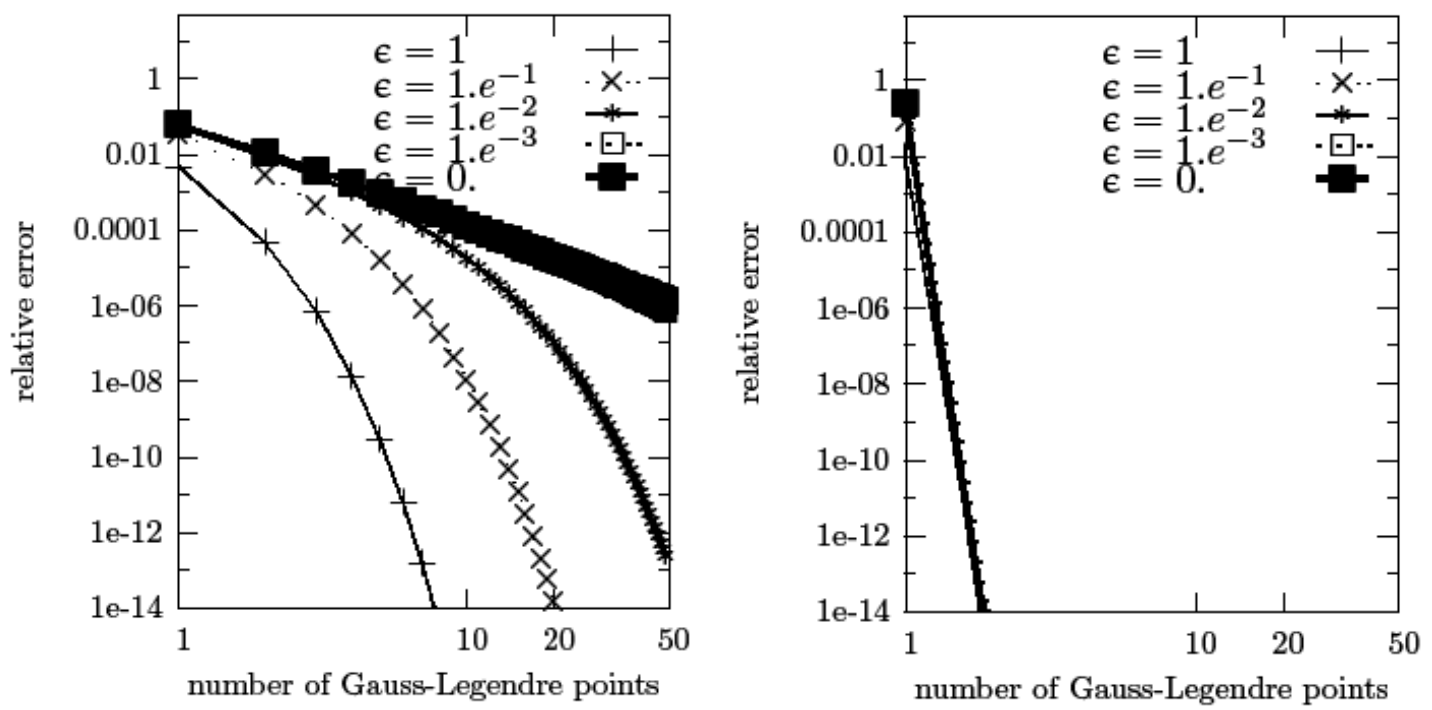

FIG. 4: Integration error of $\sqrt{(\|r\|)}$ over $[\epsilon, 1+\epsilon]$ : (left) direct Gauss-Legendre integration, and (right) GaussLegendre integration after change of variable (2.1).

Legendre points used for $\epsilon$ ranging from 1 to 0 . For large $\epsilon$, the quadrature rule converges exponentially with the number of Gauss points, but as $\epsilon$ goes to 0 the error becomes higher and higher for the same number of Gauss points. At the limit when $\epsilon$ goes to 0 , the exponential convergence is lost and a very slow power law rate is obtained. The important point that we want to make is that in fact, as long as $\epsilon>0$ the convergence is indeed exponential, but as $\epsilon$ becomes smaller, one needs more and more points to see the effect of the exponential convergence. It appears linear. Of course the integral can be computed after a change of variable $r=y^{2}$ so that the integrand becomes regular on the full integration interval:

$$
\int_{a}^{b} \sqrt{r} d r=\int_{\sqrt{a}}^{\sqrt{b}} 2 y^{2} d y
$$

Numerical integration on the right-hand side then provides excellent results, since the exact result, up to round-off error, can be obtained with the two points Gauss-Legendre rule (see Fig. 4, right).

The difficulty in integrating the term of the bilinear form associated with the crack tip enrichment functions was of course noted in numerous papers (Béchet et al., 2005; Mousavi and Sukumar, 2010; Park et al., 2009) and are founded on a change of variable that cancels the singularity. But except in Béchet et al. (2005), only the cases where the singularity is at an integration cell vertex (and/or along an edge of an integration cell in three dimensions) are treated. In Béchet et al. (2005), a solution is proposed: a triangular cell is replaced by a superposition of three cells. Each of the cells has one of its nodes on the singular point, and the two others are two nodes of the original cell. The paper reports good results in this case, but this strategy implies the use of evaluation points that are outside of the original element, which limits its application and increases the total number of evaluation points. Outside the last mentioned strategy, we found no special treatment in the X-FEM or G-FEM literature applied to cells close to the crack tip, which do not contain the crack tip. If nothing special is done for those cells, the quadrature will converge very slowly. We propose here a new integration scheme, also based on a change of variable, but we extend it to the case where the crack tip does not coincide with a node of the cell.

In order to benchmark different integration strategies, in a first step we compute the stiffness matrix of one twodimensional element and compare it with a reference solution. Let $\mathbf{K}_{i}^{e}$ be the stiffness associated with element $e$ obtained using an integration strategy, and $\mathbf{K}_{r}^{e}$ be the reference matrix. In the following we use err as our error measure, where $\operatorname{err}=\left(\left\|\mathbf{K}_{i}^{e}-\mathbf{K}_{r}^{e}\right\|_{\infty}\right) /\left(\left\|\mathbf{K}_{r}^{e}\right\|\right)$ and $\|\mathbf{A}\|_{\infty}=\max \left\{\left|a_{i j}\right|\right\}$. The reference matrix is obtained using an 
adaptive integration scheme, built on top of the function gs 1 _integration_qags provided by the Gnu Scientific Library (GSL) (Galassi, 2009). This function uses an adaptive algorithm to integrate a scalar function over an interval, until the estimated error is less than a threshold provided by the user.

\subsection{Partition of the Elements}

Starting with early X-FEM papers (Moës et al., 1999), the elements are cut along the crack front into a partition of cells that does not cross the discontinuity, and then Gauss-like integration rules over the triangular cells are used. In this part we want to discuss the splitting strategies that produce the integration cells that form a partition of the elements so that each cell is in one of the quadrant of the crack coordinate system. This is the analog to what was just presented in our first improvement of the one-dimensional case.

In the present work, we use the integration scheme over the triangle as can be found in Solin et al. (2004), where optimal rules over the triangle are given, classified by the polynomial order they can integrate exactly. We only used among these rules those which do not have negative weight nor point outside of the reference element. Table 1 gives the number of integration points for each polynomial order. For all the following integration benchmarks, the coordinates of the triangle nodes are given with respect to the crack tip coordinate system. The first results we want to discuss are results for which the crack tip are inside the element (case 1 in Table 2). The element is partitioned along the crack as in Fig. 5, according to $l s_{n}$ for case $1 \mathrm{a}$ (Fig. 5, left) and according to $l s_{n}$ and $l s_{t}$ for case $1 b$. In case $1 \mathrm{~b}$, the element is first divided in three cells by triangulating the element, after a cut along $l s_{n}=0$, then each cell of the first partition is

TABLE 1: Number of integration points (nbpt) as a function of highest-order polynomial integrated exactly over a triangle, following Solin et al. (2004)

\begin{tabular}{|c|c|c|c|c|c|c|c|c|c|c|c|c|c|c|}
\hline Order & 1 & 2 & 3 & 4 & 5 & 6 & 8 & 9 & 10 & 12 & 13 & 14 & 17 & 19 \\
\hline nbpt & 1 & 3 & 4 & 6 & 7 & 12 & 16 & 19 & 25 & 33 & 37 & 42 & 62 & 73 \\
\hline
\end{tabular}

TABLE 2: List of case tested for the different integration rule

\begin{tabular}{|c|c|c|c|c|c|c|}
\hline Case & $x 0$ & $y 0$ & $x 1$ & $y 1$ & $x 2$ & $y 2$ \\
\hline Case 1 & $-1 / 3$ & $-1 / 3$ & $2 / 3$ & $-1 / 3$ & $-1 / 3$ & $2 / 3$ \\
\hline Case 2 & 0 & 0 & 1 & 0 & 0 & 1 \\
\hline Case 3 & 0 & 0 & 10 & 0 & 0 & 1 \\
\hline Case 4 & 0 & 0 & 100 & 0 & 0 & 1 \\
\hline
\end{tabular}

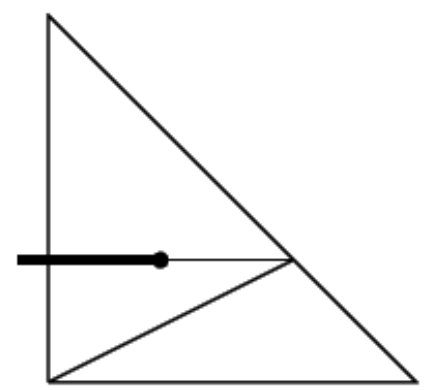

(1) cut along $l_{s_{n}}=0$

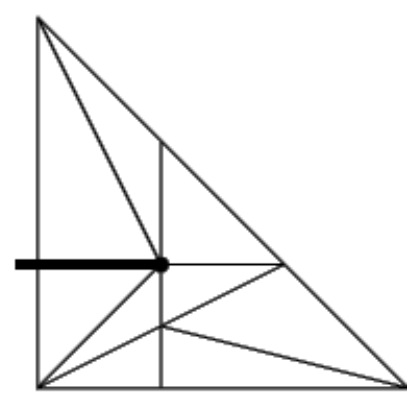

(2) cut along $l s_{n}=0$, then $l s_{t}=0$

FIG. 5: Partition of an element in an integration cell. Thick line: the crack. Black dot: the crack tip. 
further triangulated after a cut along $l_{\mathrm{t}}=0$. The goal here is to divide the element so that each cell is forced inside one of the quadrants of the crack coordinate system. Of course, this partitioning strategy is not the optimal one to achieve these properties, but it has the advantage to be built around the preexisting cut primitive to cut along the iso-zero of one level set. In case $1 \mathrm{~b}$, we therefore end up with a total of nine integration cells. With the last splitting strategy, a cell belonging to an enriched element can touch the crack tip with one of its nodes. In this case we call it a singular cell, or the crack tip is strictly outside the cell and we call this cell a weakly singular cell.

Error in the local stiffness matrix, as defined above, is plotted as a function of the total number of integration points over all the cells for the two cutting strategies as reported in Fig. 6 [For example, for the order 1 rule (one point per cell), we have three points in case 1a and nine points in case 1b]. In both cases, the convergence rate is slow. In case 1a (one cut), the convergence is not even guarantied; one can see that the error oscillates from one integration degree to the next. Even if the cut was not optimal (we integrate over nine cells), the error in case $2 b$ is constantly less than in the case of one cut, at equal number of points. The convergence, while still slow in case $1 \mathrm{~b}$, oscillates much less than for case 1a. The previous experiment has been repeated for different relative positions of the crack tip and various element shapes, and it always shows an improvement when the elements are cut along $1 s_{n}=0$ and $l s_{t}=0$. This is related to the fact that by cutting the element twice, the singularity can only be on a vertex or strictly outside a cell. In the following, the integration algorithm using the second partitioning strategy and the regular Gauss-like integration rule over the resulting triangle is called partition integration and is parametrized by the order of the integration rule $o$ used for each cell.

\subsection{Treating the Singularity}

As seen above, partitioning the element into a suitable partition of cells improves the situation by confining the singularity on a corner of a cell. For all elements in the enrichment zone, there are terms in the form of $1 / r, 1 / \sqrt{(r)}$, $\sqrt{(r)}, r$ which are singular or singular in their gradient for $r=0$. In the literature, such singularity is already treated properly for a cell which has one node on the singularity. In most cases, the authors used changes of variables before performing the integration that renders the integrand regular. Among those changes of variables, we concentrate on the one proposed in Béchet et al. (2005). First, a cell with a node on the crack tip is mapped via a linear mapping to a reference element that has the crack tip on its first node. Let us label $\xi$ and $\eta$ the coordinates in the reference element. Then the following change of variable is applied:

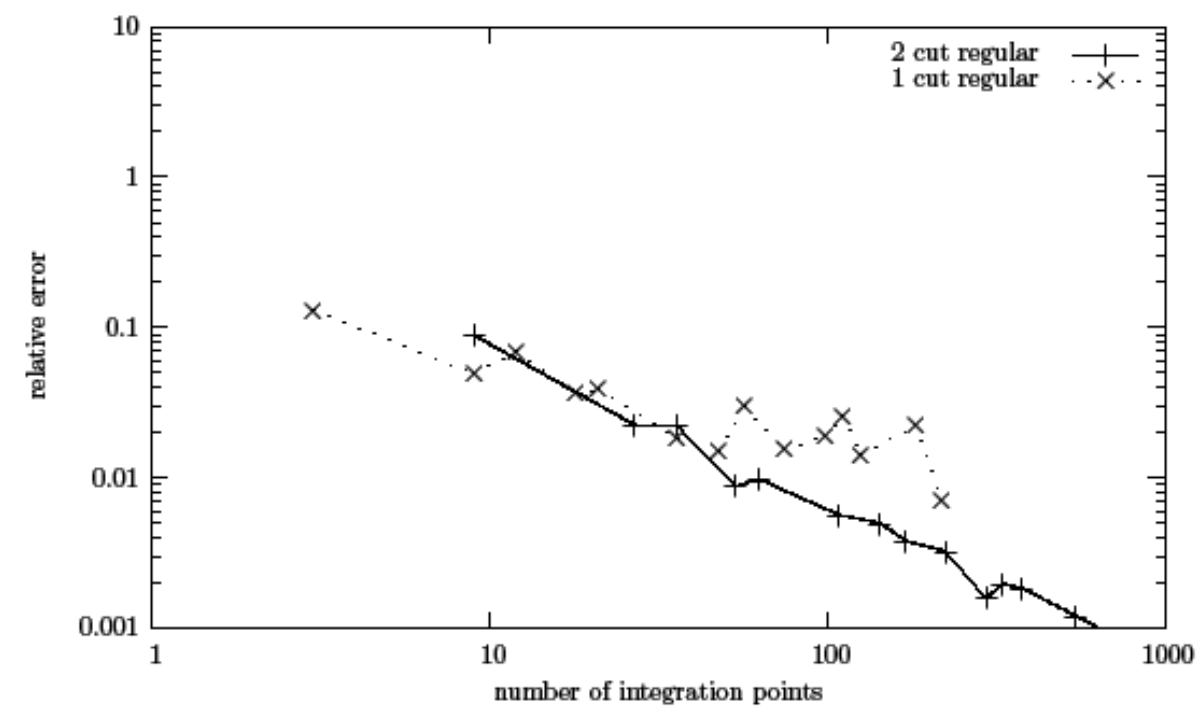

FIG. 6: Max norm of relative stiffness matrix error for case 1, with one or two cuts. 


$$
\xi=\frac{1}{2} \rho^{2}[1-\sinh (\tau)], \quad \eta=\frac{1}{2} \rho^{2}[1+\sinh (\tau)]
$$

where $\rho$ takes values in $[0: 1]$ and $\tau$ in $\{-\ln [1+\sqrt{2}]: \ln [1+\sqrt{2}]\}$. The change of variable defined in Eq. (2.17) can be seen as a map between the rectangle $\{[0,-\ln (1+\sqrt{2})],[1,-\ln (1+\sqrt{2})],[1,+\ln (1+\sqrt{2})],[1,+\ln (1+\sqrt{2})]\}$ in the $(\rho, \tau)$ plane to the triangle $[(0,0),(1,0),(0,1)]$ in the $(\xi, \eta)$ plane, as represented in Fig. 7 . Note that the grid represented in the triangle is made of the isolines of $\rho$ and $\tau$. The singular point $A$ in the triangle is the image of the line $\rho=0$. This change of variable is very close to a polar change of variable, where $\tau$ plays the role of the angle and where $\rho$ is close to the square root of the radius.

This change of variable was built with the previous consideration in mind and so that the domain that represents the triangle in the $\rho, \tau$ plane is a simple rectangle, where it is easy to build an integration rule by using a tensor product of Gauss-Legendre rule in each direction of the $(\rho, \tau)$ plane. This is exactly what is done in our next numerical experiment. We integrate the stiffness matrix for an element which corresponds to the reference element with the singularity at the first node (case 2 in Table 2), for different numbers of Gauss-Legendre points using the presented change of variable, and compare using regular integration points over the triangle. The one-dimensional Gauss-Legendre rules used to build the two-dimensional rules are rules starting at one point and ending at ten points. The number of integration points for the two-dimensional rules therefore range from 1 to 100 .

Figure 8 reports the error in the stiffness matrix as a function of the total number of Gauss points. The results are of course much better using the change of variables. We can observe an exponential convergence rate. The error reaches a value of $7.28 \times 10^{-9}$ for 81 points and $7.406 \times 10^{-10}$ for 100 points, while the classic integration rule of highest order on the triangle just reaches $9.77 \times 10^{-3}$ with 79 points.

Unfortunately, even for the case where one node is exactly singular, the results are not always good enough. Even if the change of variable always improves the situation, the fact that the change of variable is done with regard to a reference element in order to always have a known rectangular domain on which to develop the integration rule can induce a relative loss of efficiency when the cell on which one wishes to integrate has a high aspect ratio (the shortest edge is much smaller than the longest edge), as can be seen in the following experiment. Cases 2, 3, and 4 from Table 1 are tested; they are all elements with the first node at the singularity, the third node at $(0,1)$, but the second node is at $y=0$, and $x$ is respectively set to $L=1, L=10$, and $L=100$. The goal of the experiment is here to relate the aspect ratio of the cell to the accuracy of the integration rule. As can be seen in Fig. 9, the quality of the

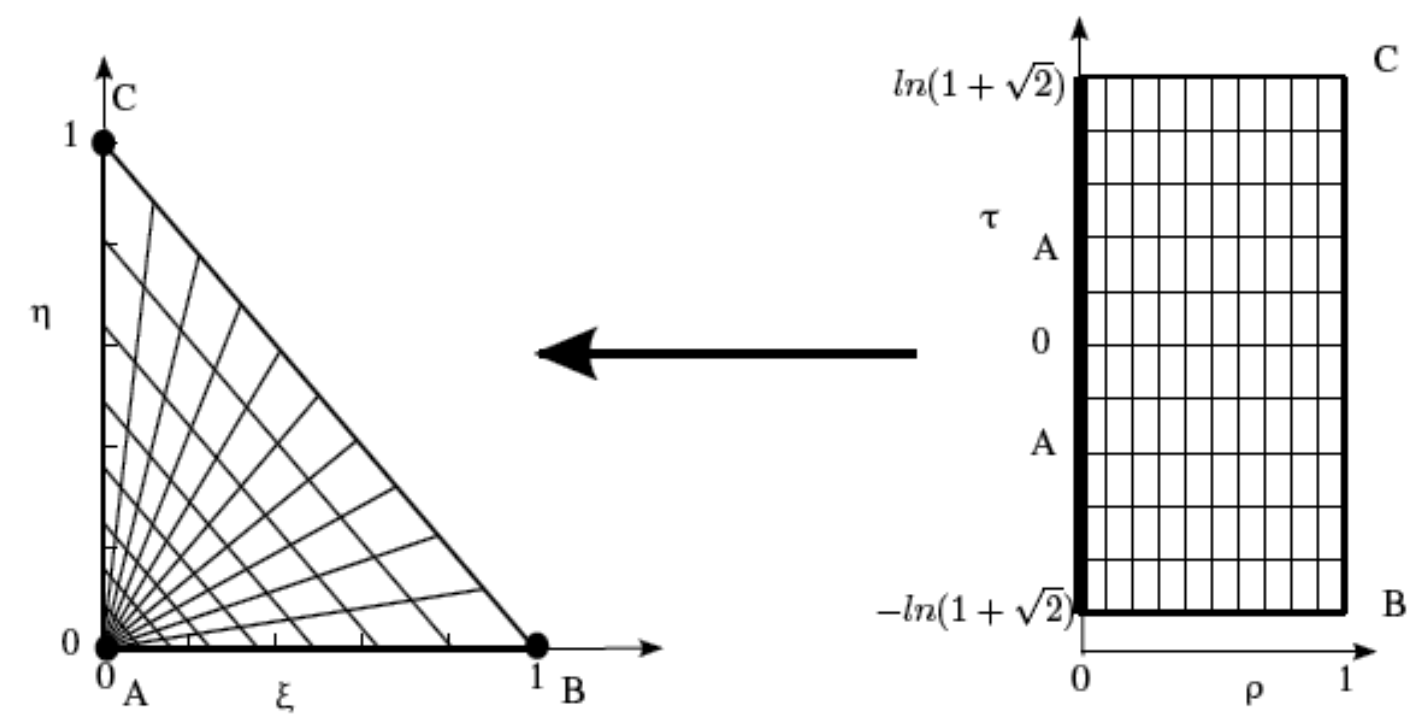

FIG. 7: Change of variable according to Eq. (2.17) for a cell with a singular node. 


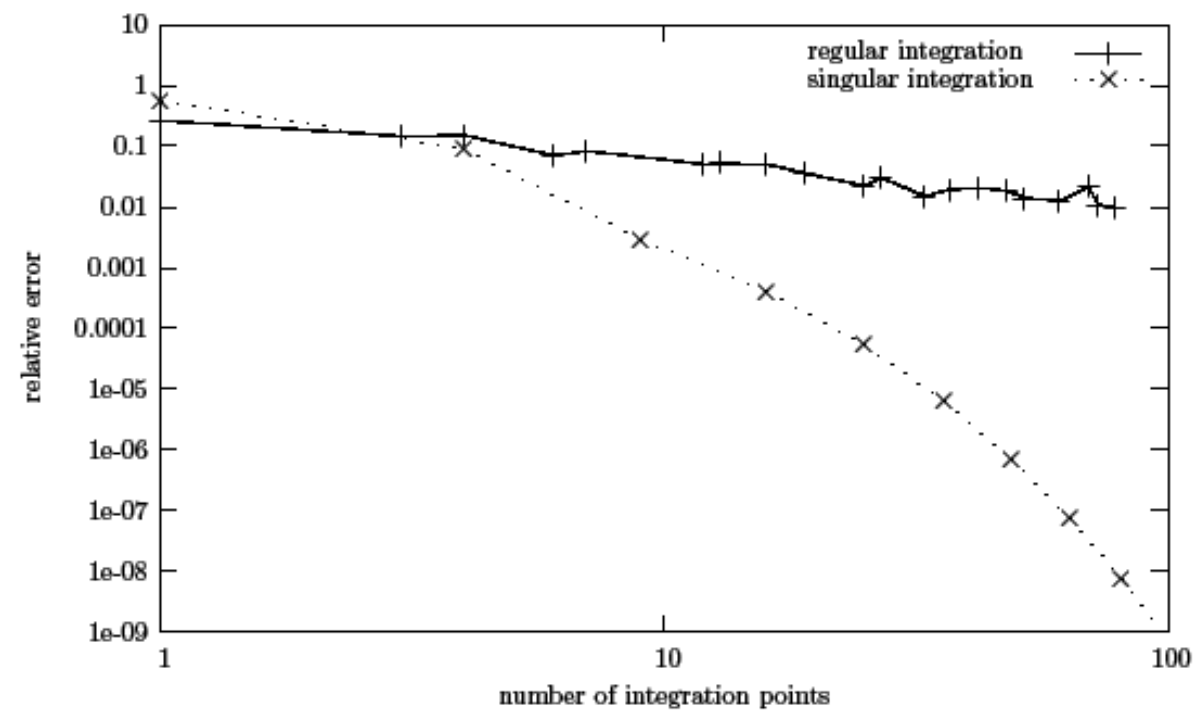

FIG. 8: Error in stiffness matrix max norm for case 2, comparing regular integration with Gauss-Legendre integration using a change of variable 2.17 .

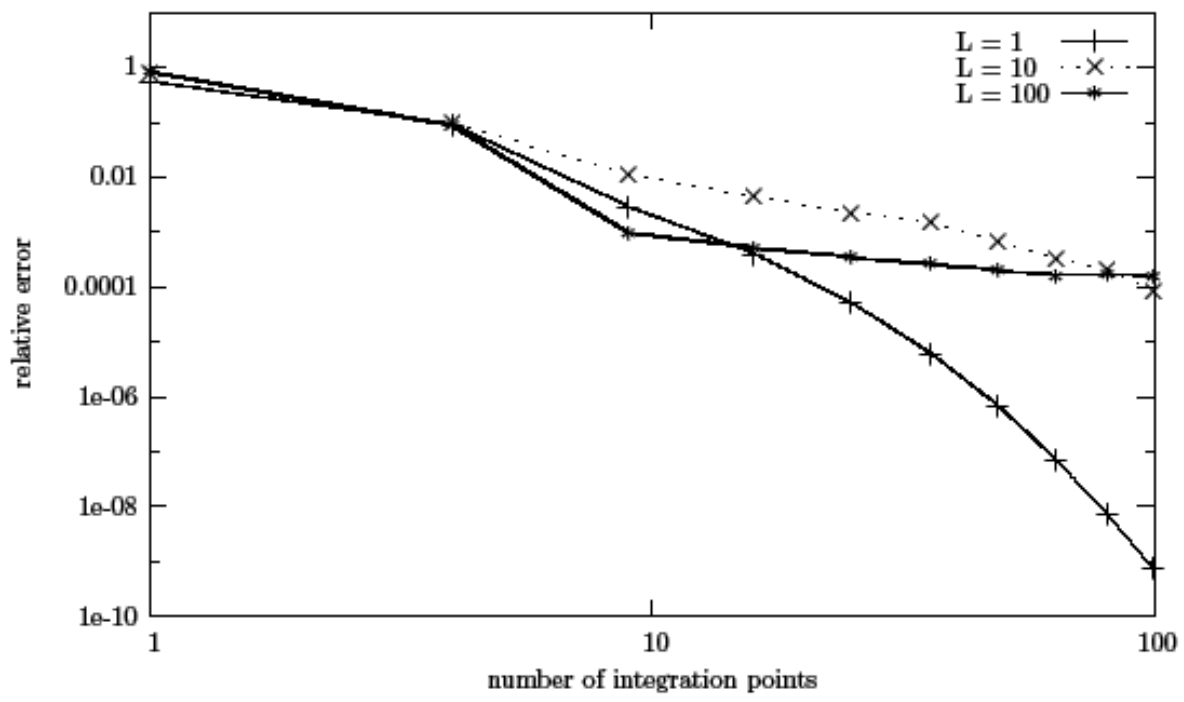

FIG. 9: Error in Stiffness matrix max norm for cases 2, 3, and 4: with a varying aspect ratio $\max (x) / \max (y)$ using change of variable 2.17 .

convergence is clearly degraded by the high aspect ratio. This point is, in fact, very important for the robustness of the overall method. Indeed, since the mesh is not related to the crack, the positions of the nodes relative to the crack type will dictate the shape of the integration cells. Even with a very-high-quality mesh, the cells can have a very bad aspect ratio. It happens if one node of the mesh is very close to the crack surface. Therefore, even for the case where one cell node is on the crack tip, the strategy proposed in most papers suffers from robustness issues even when using a special integration scheme of the type we just presented. But the situation in the particular case of a node on the crack tip, as we will show, is going to be improved by the general approach that we develop in the following. 


\subsection{Treating the Singularity for All Enriched Cells: the Algorithm}

We have just shown that even when one node of a cell is on the crack tip, singular integration schemes are insufficient to get a rapid and robust convergence of the integration of the stiffness matrix. But perhaps more importantly, we also need to consider the case where the crack tip is outside of the element but still close to the element. This case is analogous to our one-dimensional analysis where the singularity was outside of the integration domain: even if the singularity is outside, the convergence rate is very slow if no change of variable is applied. So far, all the integration schemes that we used were found in the literature. In this section, we develop what we think is a new contribution to the field. After the discussion about the partition of the elements, we place ourselves in a framework where a cell is always in one of the quadrants of the crack coordinate system. Furthermore, we propose that a cell has to be in the positive quadrant $(x \geq 0$ and $y \geq 0$ ). If this is not the case, the cell is first mapped to the positive quadrant by reversing one or two of its node coordinates. No further mapping to a reference element is applied, contrary to the scheme presented in the previous section. Indeed, since the singular point can be outside of the element, there is no gain to apply any such mapping. We then consider the same change of variable but directly applied to a triangle in crack the coordinate system (positive quadrant).

$$
x=\frac{1}{2} \rho^{2}[1-\sinh (\tau)], \quad y=\frac{1}{2} \rho^{2}[1+\sinh (\tau)]
$$

The domain that represents the triangle in the $(\rho, \tau)$ plane can have a variety of shapes. If no node is on the crack tip, the shape in the $(\rho, \tau)$ plane is a curved triangle. When a node of the triangle is continuously moved to the crack tip, the curved triangle looks more and more like a quadrilateral with one straight edge on $\rho=0$ at the end of the motion. Figure 10 gives some examples of the effect of mapping for some typical cell.

We now wish to integrate the stiffness matrix after having performed the change of variable by mapping GaussLegendre points on the unit square to the triangle in the $(\rho, \tau)$ plane. Fortunately, in order to perform this task, we can count on some properties of the change of variable. First, the change of variable can be inverted for all points but the
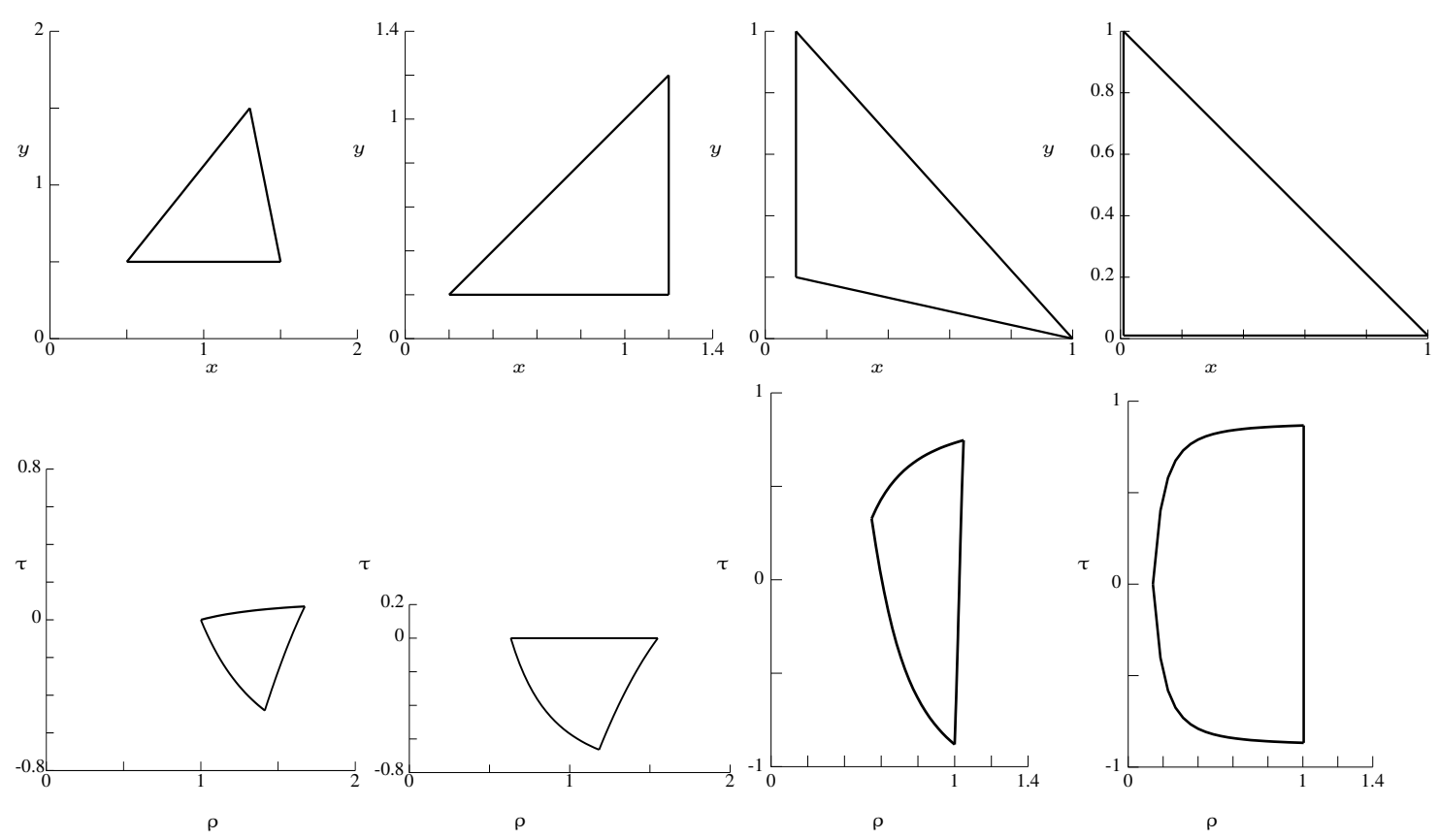

FIG. 10: Effect of the change of variable (2.3) on some cells. Top: cell in the $(x, y)$ plane, Bottom: corresponding cell in the $(\rho, \tau)$ plane. 
singular point, which means that by knowing the coordinate $x, y$ of a point in the $(x, y)$ plane, the coordinates $\rho, \tau$ can be computed as

$$
\psi^{-1}:(x, y) \rightarrow(\rho, \tau) \quad x \geq 0 \quad y \geq 0 \quad x+y>0, \quad \rho=\sqrt{x+y}, \quad \tau=\operatorname{arcsinh}\left(\frac{y-x}{x+y}\right)
$$

Let us now consider a segment $[A B]$ in the $(x, y)$ plane. This segment is mapped on a curve on the $(\rho, \tau)$ plane, for which the graph and its derivative can be computed. Let $\alpha_{A B}, \beta_{A B}$, and $\gamma_{A B}$ be three constant values computed as follows from the coordinates $x_{A}, y_{A}$ and $x_{B}, y_{B}$ :

$$
\begin{gathered}
\alpha_{A B}=\left(x_{A}+x_{B}\right)\left(y_{B}-y_{A}\right)-\left(y_{A}+y_{B}\right)\left(x_{B}-x_{A}\right) \\
\beta_{A B}=x_{A}-x_{B}-y_{A}+y_{B} \\
\gamma_{A B}=x_{A}-x_{B}+y_{A}-y_{B}
\end{gathered}
$$

The segment $[A B]$ is described by the following curve $\rho_{A B}(\tau)$ and derivative $\rho_{A B}^{\prime}(\tau)$ in the $(\rho, \tau)$ plane, if $\tau_{A} \neq \tau_{B}$ :

$$
\begin{gathered}
\rho_{A B}=\sqrt{\frac{\alpha_{A B}}{\beta_{A B}+\gamma_{A B} \sinh (\tau)}} \\
\rho_{A B}^{\prime}=-\frac{1}{2 \alpha_{A B}} \rho^{3} \gamma_{A B} \cosh (\tau)
\end{gathered}
$$

Alternatively, it can be described by the curve $\tau(\rho)$ and derivative $\tau^{\prime}(\rho)$ if $\rho_{A} \neq \rho_{B}$ :

$$
\begin{gathered}
\tau_{A B}=\operatorname{asinh}\left(-\beta_{A B} / \alpha_{A B}+\alpha_{A B} / \beta_{A B} \rho^{-2}\right) \\
\tau_{A B}^{\prime}=\frac{-2 \alpha_{A B}}{\beta_{A B} \rho^{3}} \frac{1}{\sqrt{1+\left(-\beta_{A B} / \alpha_{A B}+\alpha_{A B} / \beta_{A B} \rho^{-2}\right)^{2}}}
\end{gathered}
$$

An important property of the curve $\rho_{A B}(\tau)$ is that it is monotonous in $\tau$ for all points of the segment $[A B]$ in the positive quadrant [respectively $\tau_{A B}(\rho)$ is monotonous in $\rho$ ]. It means that the curve representing one edge of a triangle in the $(\rho, \tau)$ plane is increasing or decreasing, exclusively. This property will be used very shortly.

In a first step of our algorithm to build an integration rule over the image of the triangle in the $(\rho, \tau)$ plane, we first classify each edge $[A B]$ according to the value of $\tau_{A B}^{\prime}(\rho)$ along the edge. If locally $\tau_{A B}^{\prime}(\rho)$ is in $[-1: 1]$, then the tangent of the curve is labeled to be "along" the $\rho$ direction; otherwise it is labeled to be along the $\tau$ direction. Since the curve for one edge is monotonous, only four situations can occur for the "direction" of the edge, depending on the value of $\tau_{A B}^{\prime}(\rho)$ at the extremities $A$ and $B$ of the segment:

i. $\left(\tau_{A B}^{\prime}\left(\rho_{A}\right) \in[-1: 1]\right)$ and $\left(\tau_{A B}^{\prime}\left(\rho_{B}\right) \in[-1: 1]\right)$

ii. $\left(\tau_{A B}^{\prime}\left(\rho_{A}\right) \notin[-1: 1]\right)$ and $\left(\tau_{A B}^{\prime}\left(\rho_{B}\right) \notin[-1: 1]\right)$

iii. $\left(\tau_{A B}^{\prime}\left(\rho_{A}\right) \in[-1: 1]\right)$ and $\left(\tau_{A B}^{\prime}\left(\rho_{B}\right) \notin[-1: 1]\right)$

iv. $\left(\tau_{A B}^{\prime}\left(\rho_{A}\right) \notin[-1: 1]\right)$ and $\left(\tau_{A B}^{\prime}\left(\rho_{B}\right) \in[-1: 1]\right)$ 
In case 1 , the edge is said to be in the $\rho$ direction, and in case 2 , the edge is said to be in the $\tau$ direction. In cases 3 and 4 , there exists one and only one point $a b$ on the edge $[A B]$, such as we have $\tau_{A B}^{\prime}\left(\rho_{a b}\right)=1$ or $\tau_{A B}^{\prime}\left(\rho_{a b}\right)=-1$. We call this point a cut point, and it can be found easily by using any root solver. In such a case, the edge is split in two parts, each part with one properly defined direction, either $\rho$ or $\tau$. Therefore for any triangle, by eventually splitting each edge at most once, we can construct a list of at most six edge parts. Each edge part is aligned either in the $\tau$ or $\rho$ direction. A number of cases depending on the alignment of each edge part in the $(\rho, \tau)$ plane can be recognized, but before pursuing, let us include the case where one node of the cell is on the singularity. In this case, the mapping from the $(\rho, \tau)$ plane to the $(x, y)$ plane is not invertible for points on the line $\rho=0$. The image of the singular point of a triangle is a segment in the $(\rho, \tau)$ plane along the $\rho=0$ axis and its direction is $\tau$. The two edges connecting the singular point produce two segments for which $\tau$ is fixed and therefore, their direction is $\rho$. Only the image of the third edge of the triangle is curved on the $(\rho, \tau)$ plane. This edge cannot have any singular point, and the previous formulas can be used with no change to choose its direction or split it and choose the direction of its edge parts if needed (see Fig. 11). We end up with a list of at most four edge parts in this case, and the algorithm beyond this point is the same for cells with or without one node on the singular point. To keep the discussion brief, let us first discuss a typical case as seen in Fig. 12. The triangle $(A B C)$ is mapped in the $(\rho, \tau)$ plane. According to our splitting rule, the edge $[A B]$ is split in $[A a b]$, which is found to be in the $\tau$ direction, and $[a b B]$, which is in the $\rho$ direction. The edge $[A C]$ is split in $[A c a]$, which is in the $\tau$ direction, and $[c a C]$, which is in the $\rho$ direction. From these five edges, we now want to construct a partition of the image of the triangle in the $(\rho, \tau)$ plane so that each subcell is a curved quadrilateral (or eventually curved quadrilateral degenerated to a curved triangle when two consecutive nodes are at the same position). But we do not want any curved quadrilateral, we want quadrilateral, so that when traversing the contour-connected curved edge by the connected curved edge, the direction of edge 1 is $\rho$, edge 2 is $\tau$, edge 3 is $\rho$, and edge 4 is $\tau$. By imposing these conditions, we end up with a curved quadrilateral, with each edge as much as possible aligned with the $\rho, \tau$ coordinate axis. In the case presented in Fig. 12, it is possible to obtain a partition in two quadrilaterals by constructing one more point: the point $[b c]$, which is the intersection of the curve $[B C]$ with the axis $\tau=\tau_{A}$. Note that this point is easy to obtain because we have an explicit equation for the curve $[B C]$ in the form of the $\rho_{B C}(\tau)$. The two curved quadrilaterals, respecting the direction condition and forming a partition of the
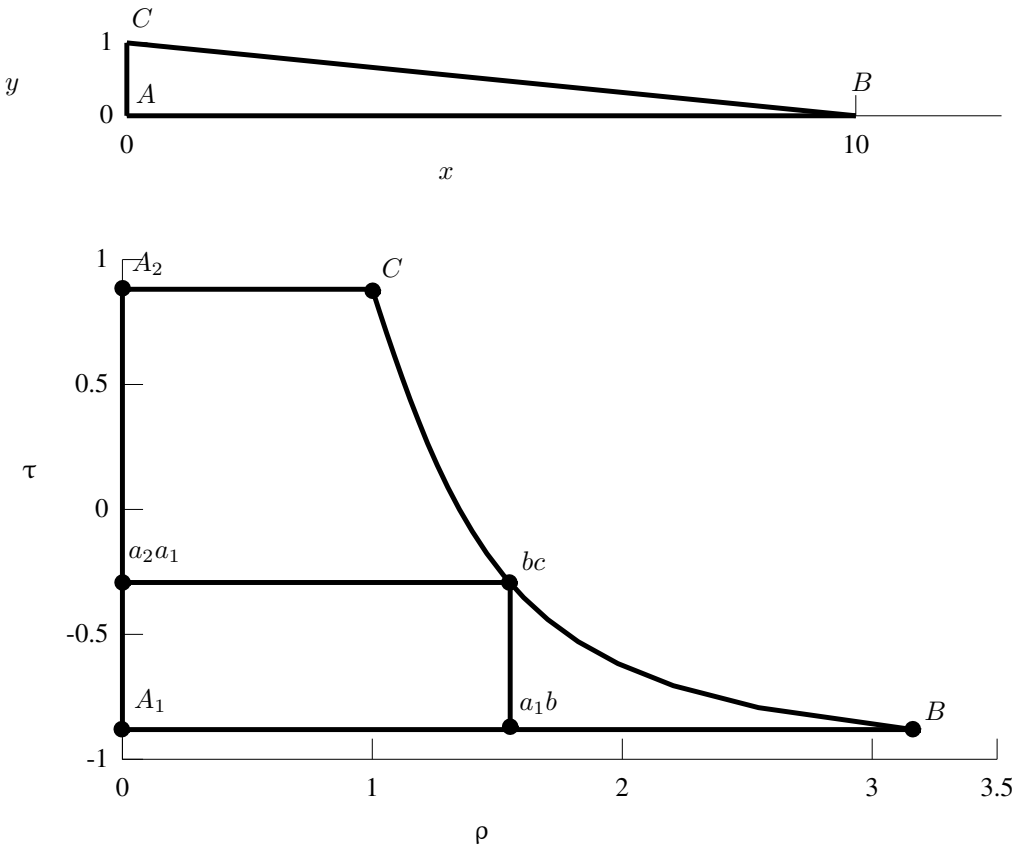

FIG. 11: Mapping for element of case 3 [top in $(x, y)$ plane] with subcell splitting in $(\rho, \tau)$ plane (bottom). 


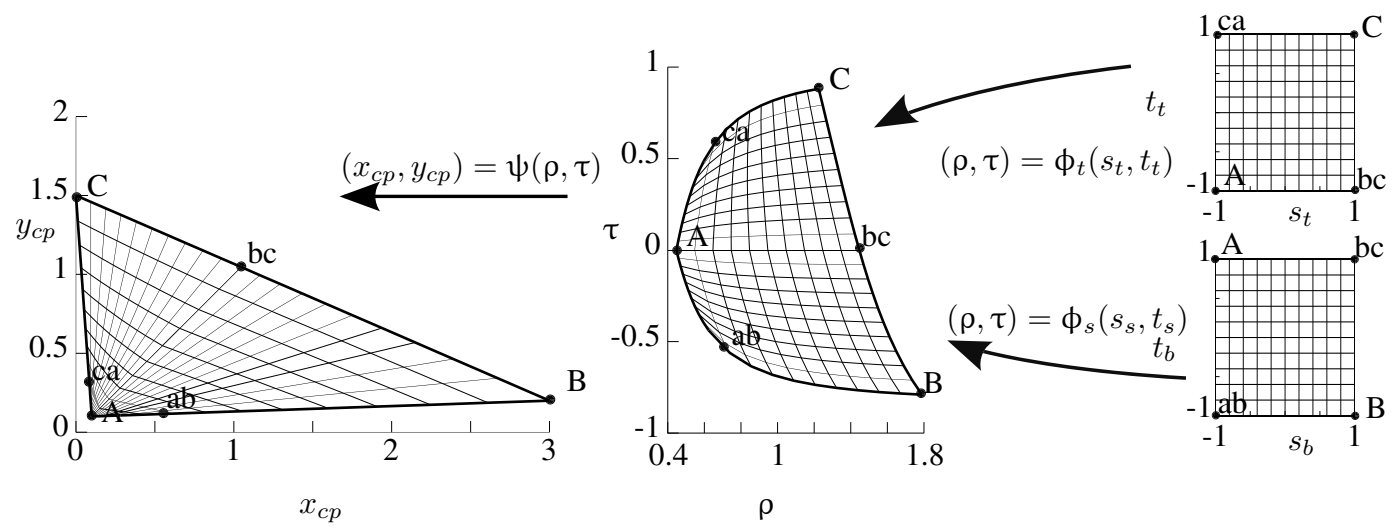

FIG. 12: Singular integration scheme.

image of $[A B C]$, are now $[A b c C c a]$ and $[a b B b c A]$. Each of these curved quadrilaterals can now be mapped on a reference square in the $(s, t)$ plane. The details of the subcell construction algorithm for all the different cases is quite technical, would take a lot of space, and are not necessary to pursue the discussion. We prefer to directly distribute the code, which will be freely available upon request.

In the following, we call $\left[A^{\prime} B^{\prime} C^{\prime} D^{\prime}\right]$ a curved quadrilateral in the $(\rho, \tau)$ plane, with edge $\left[A^{\prime} B^{\prime}\right],\left[B^{\prime} C^{\prime}\right],\left[C^{\prime} D^{\prime}\right]$, and $\left[D^{\prime} A^{\prime}\right]$ respectively in the $\rho, \tau, \rho$, and $\tau$ direction, with the the previously defined meaning. This quadrilateral is mapped to a reference square in the $(s, t)$ plane, such as $A^{\prime}, B^{\prime}, C^{\prime}$, and $D^{\prime}$ are, respectively, the image of $(-1,-1)$, $(1,-1),(1,1)$, and $(-1,1)$ by

$$
\begin{aligned}
& \rho=\frac{1}{2}(1-s) \rho_{A^{\prime} D^{\prime}}(t)+\frac{1}{2}(1+s) \rho_{B^{\prime} C^{\prime}}(t) \\
& \tau=\frac{1}{2}(1-t) \tau_{A^{\prime} B^{\prime}}(s)+\frac{1}{2}(1+t) \tau_{D^{\prime} C^{\prime}}(s)
\end{aligned}
$$

where the functions $\rho_{A^{\prime} D^{\prime}}(t), \rho_{B^{\prime} C^{\prime}}(t), \tau_{A^{\prime} B^{\prime}}(s)$, and $\tau_{D^{\prime} C^{\prime}}(s)$ need to be defined. We detail the construction of $\tau_{A^{\prime} B^{\prime}}(t)$ in the following; the three other functions are constructed in a similar way. First note that by construction, edge $\left[A^{\prime} B^{\prime}\right]$ is aligned with $\rho$, following our definition $-\tau_{A^{\prime} B^{\prime}}^{\prime}(\rho)$ is in $[-1: 1]$ and cannot change sign being either positive, negative, or identically zero. If the slope is identically zero, then $\tau_{A^{\prime} B^{\prime}}(s)=\tau_{A^{\prime}}=\tau_{B^{\prime}}$. Otherwise, we first define $\rho_{A^{\prime} B^{\prime}}(s)$ by mapping linearly $[-1: 1]$ to $\left[\rho_{A^{\prime}}: \rho_{B^{\prime}}\right]$. Then we set $\tau_{A^{\prime} B^{\prime}}(s)=\tau_{A^{\prime} B^{\prime}}\left[\rho_{A^{\prime} B^{\prime}}(s)\right]$. This is of course always possible since the curve is monotonous. This mapping is reproduced in Fig. 12. A square in the $(s, t)$ is mapped on each of the curved quadrilateral subcell of the image of $(A B C)$ in the $(\rho, \tau)$ plane. On this figure the grid is defined on the square of the $(s, t)$ plane and is successively mapped on the $(\rho, \tau)$ and $(x, y)$ plane, from right to left. It is now easy to construct an integration rule: For each quadrilateral in the $(s, t)$ plane that forms a valid partition of a cell, construct a Gauss-Legendre rule which is a tensor product of classic one-dimensional rule. Then compute the coordinate of each point in the $(x, y)$ plane by chaining the mapping and the change of variable. The overall algorithm can be summarized as follows, for a given triangular cell:

i. Compute the direction of each edge in the $(\rho, \tau)$ plane.

ii. Split edges that need it at the point $\tau^{\prime}(\rho)=+1$ or -1 .

iii. Divide the cell in "aligned" curved quadrilateral.

iv. For each quadrilateral, construct a Gauss-Legendre integration rule using the mapping between the $(s, t)$ and the $(\rho, \tau)$ plane. 
Each of the steps of the previous algorithm, except for the second one, have been fully described. There are a lot of possible alternatives to achieve this second step, and the implementation can be tedious. The full description of the second step as we implemented it, for the sake of the tractability of the presentation, is not exposed in the paper. The code that constructs it, as mentioned before, will be given upon request to the authors. We present and analyze results of this integration scheme in the next section.

\subsection{Treating the Singularity for All Enriched Elements: Some Results}

First we show the convergence rate for the elements presented at the beginning of the section, and then we present a more global benchmark of the integration rule that tests the algorithm for a large quantity of elements.

\subsubsection{Integration Error Over One Element}

Cases 2, 3, and 4 were designed with various degrees of aspect ratio, as previously described to measure the integration error when an element or a cell was strictly singular. Results applying the new algorithm are displayed in Fig. 13 and are to be compared with the previous results using only the change of variable presented in Fig. 13. The results for case 2, which is the reference element, are exactly the same as results obtained using only the variable change. This is because no edge split is needed in this case and the integration rule produced is exactly the same. For cases 3 and 4 , the results are greatly improved. For these two cases, the edge not connected to the crack tip is split in such a manner that the algorithm, as represented in Fig. 11 for case 3, produces three subcells aligned with the $(\rho, \tau)$ axis: $\left[A_{1}, a_{1} b, b c, a_{2} a_{1}\right],\left[a_{2} a_{1}, b c, C, A_{2}\right]$, and $\left[a_{1} b, B, B, b c\right]$ (the last quadrilateral being degenerated to a triangle). This explains the total number of integration points reaching 300 for the highest-order rule used (again, a 10-point onedimensional Gauss-Legendre rule). The best rule returns an error as low as $1.87 \times 10^{-11}$ and $3.482 \times 10^{-8}$ for cases 3 and 4, respectively, to compare with the value obtained by applying only the change of variable: $8.235 \times 10^{-5}$ and $1.521 \times 10^{-4}$, respectively.

Case 1, which was first used to compare the merit of two splitting strategies into cells, as can be seen in Fig. 5 does not produce only cells with one node on the crack tip but also produces cells that, in the second splitting strategy,

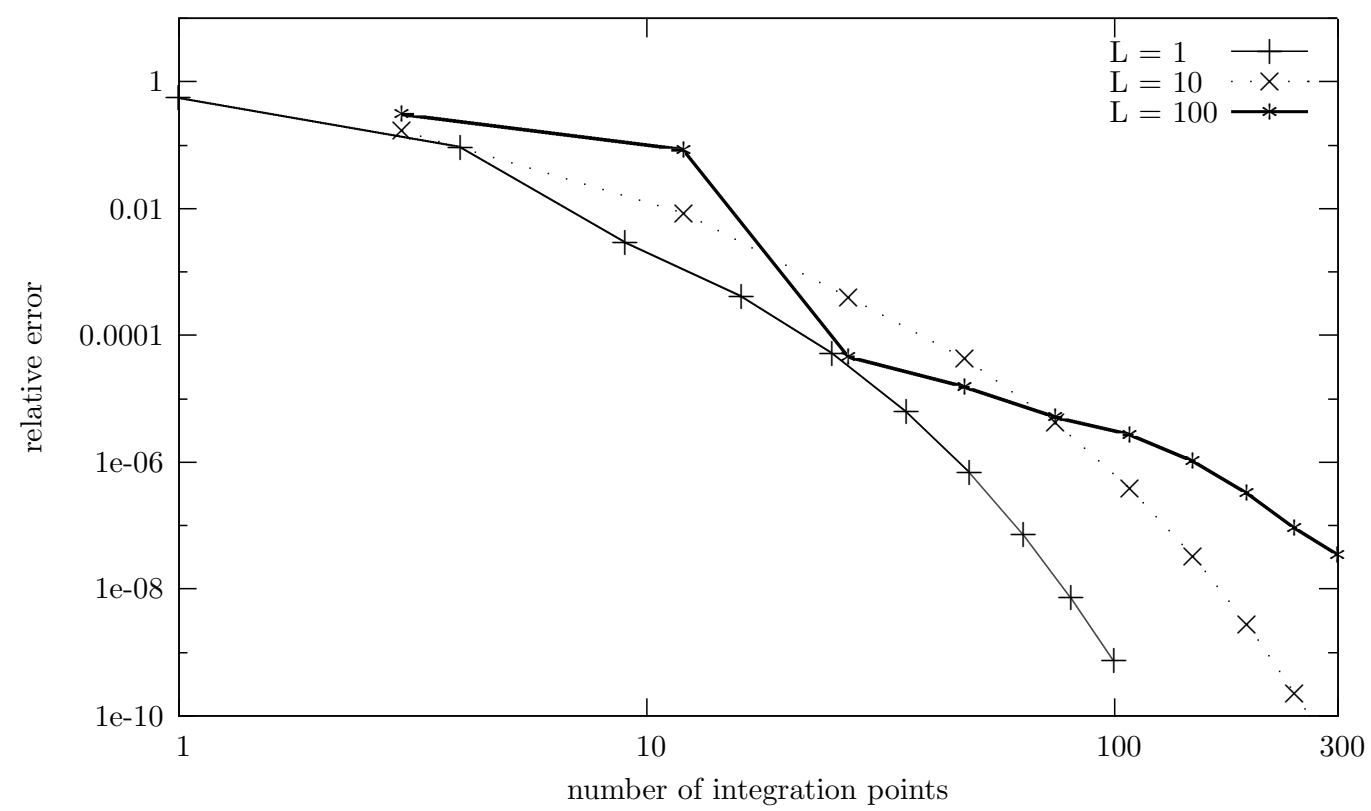

FIG. 13: Error in Stiffness matrix max norm for cases 2, 3, and 4, varying the aspect ratio $\max (x) / \max (y)$ using the new algorithm. 
have a singularity close and outside of the element. Applying only the change of variable is not practicable for those cells, but the new algorithm is applicable for all the cells. As mentioned before, the splitting strategy produces nine integration cells, and the new algorithm from these nine cells generates a total of $17(\rho, \tau)$ aligned subcells. This appears to be a lot, but as can be seen in Fig. 14, this is still an efficient algorithm: The error obtained applying the one-dimensional 10-point Gauss-Legendre rule (for a total number of 1700 points) reaches an error of $3.47 \times 10^{-9}$, while the best partition integration (for a total number of $9 \times 79=711$ integration points) only reaches $9.015 \times 10^{-4}$.

\subsubsection{Integration Error Over an Unstructured Mesh}

The experiment could be repeated for a lot of elements, with varying shapes and relative positions to the crack tip, but in the following we propose what we think is a better benchmark that tests at once for a great variety of elements. The elasticity problem is solved on a square that contains a straight horizontal crack with the crack tip at the center of the square (see Fig. 15). On the boundary of the square, the tension found by computing the stress of an exact solution for a crack in an infinite domain is applied. The exact solution of this problem coincides with the exact solution in infinite domain, inside the square. The square is meshed with an unstructured mesh, and the displacement field is discretized by using the tip enrichment everywhere, and only the tip enrichment, multiplied by the partition of unity. The only difference with classical benchmarks used to show the $h$ convergence of the X-FEM method, as used, for instance, in Béchet et al. (2005), is that we use only the tip enrichment functions, and these functions are used everywhere in the mesh. In principle, if the integration of the bilinear form was exact and if the obtained system of linear equation was not singular, we should end up with the exact solution, since the exact solution is contained in the enriched finite element space we just constructed on our square. Our first experiments trying this strategy were unsuccessful when using what we call the classic scalar enrichment in the first part of this paper. Indeed, when using a weak integration rule on quite coarse meshes, we were able to obtain some results, with quite high error, but when the mesh was refined or the integration rule was improved, we often end up with a singular system of equations that we could not solve with a direct solver (in our case superlu, described in Demmel et al. 1999). Further analysis, and as was noted in Béchet et al. (2005) or Laborde et al. (2005), showed that the space obtained by using the four scalar shape functions, produces

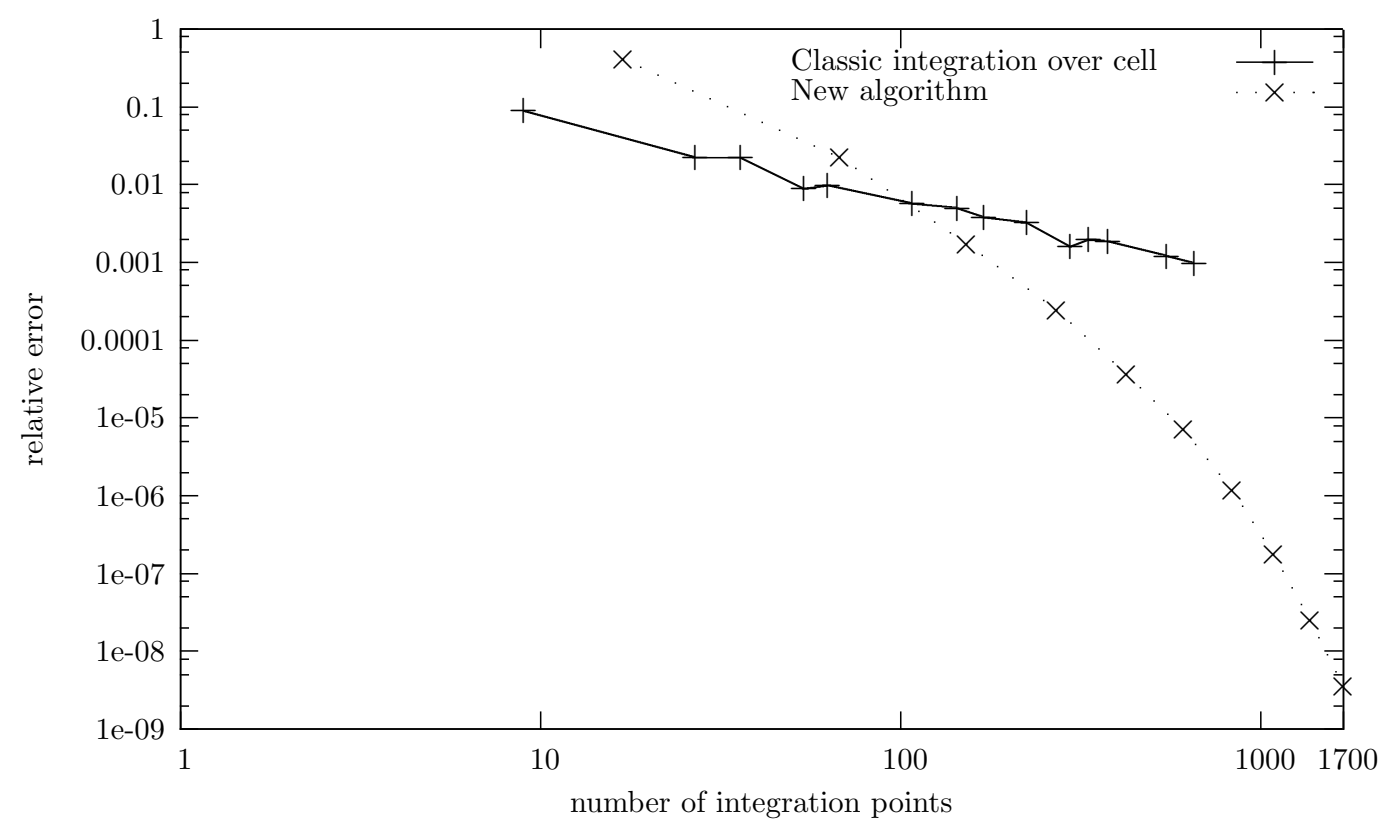

FIG. 14: Error in stiffness matrix max norm for case 1, crack tip inside the element, using regular integration and the new algorithm. 


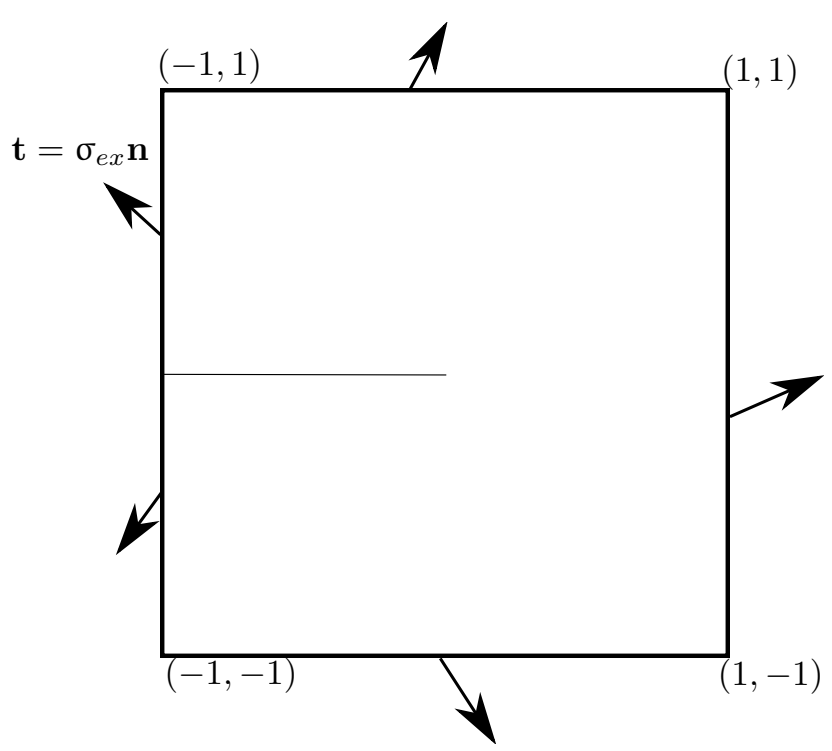

FIG. 15: Sketch of the benchmark problem. Traction on the boundary of the square (arrows) is computed from the exact solution of a straight crack in infinite media.

a more and more ill-conditioned stiffness matrix as the enrichment zone grows, to the limit of a singular matrix when the enrichment is applied on all the nodes. As will be shown, this is not the case with the vector enrichment shape function proposed in this paper. But to keep the presentation consistent, we still want to use the scalar enrichment to show the gain of an improved integration strategy. In order to do that, we will reduce the enriched space a little bit by keeping only the needed enrichment function to produce a space that still includes the pure mode I opening solution. This reduction will be used only for the present benchmark. The other experiment wil, of course, use the full scalar enrichment. The full tip enrichment space in the two-dimensional case is reminded in the following equation:

$$
\sum_{i \in[1: 2]} \sum_{I \in \mathcal{N}_{\text {tip }}} \sum_{\alpha \in[1: 4]} N_{I}(\mathbf{x}) F_{\alpha}(\mathbf{x}) \mathbf{E}_{i} a_{I \alpha i}
$$

The directions of the physical space are enriched with each of the four scalar enrichment functions. We reduce this space by using a space where only $F_{1}$ and $F_{2}$ are used in both the $\mathbf{E}_{x}$ and $\mathbf{E}_{y}$ direction, while $F_{3}$ and $F_{4}$ are only applied, respectively, in the $\mathbf{E}_{x}$ and $\mathbf{E}_{y}$ direction. The space of shape functions used in our benchmark, using scalar enrichment function everywhere, is now

$$
\sum_{I \in \mathcal{N}} N_{I} F_{1} \mathbf{E}_{x} a_{I 1 x}+N_{I} F_{1} \mathbf{E}_{y} a_{I 1 y}+N_{I} F_{2} \mathbf{E}_{x} a_{I 2 x}+N_{I} F_{2} \mathbf{E}_{y} a_{I 2 y}+N_{I} F_{3} \mathbf{E}_{x} a_{I 3 x}+N_{I} F_{4} \mathbf{E}_{y} a_{I 4 y}
$$

This reduction of the space insures the presence of a pure mode I opening field and produces a regular stiffness matrix when all the nodes of a mesh are enriched. The problem is solved on a square aligned with the crack, with crack tip at $(0,0)$, the two opposite corners of the square at position $(-1,-1)$ and $(1,1)$, respectively. The mesh is built using the Gmsh package (Geuzaine and Remacle, 2009; Remacle et al., 2007). Each edge of the square is divided in 20 equal sized segments, for a total of 560 nodes and 1038 triangles for the final unstructured mesh. With the space used, we end up with 3360 degrees of freedom, 1228 integration cells and 1805 sub-cells for the new integration strategy.

For this test, we define the error err to be the relative energy error norm defined as follows

$$
e r r=\sqrt{\int_{\Omega^{h}} \frac{\left(\sigma^{h}-\sigma^{e}\right):\left(\epsilon^{h}-\epsilon^{e}\right)}{\sigma^{e}: \epsilon^{e}} d \Omega}
$$

where $\sigma^{h}, \epsilon^{h}, \sigma^{e}$ and $\epsilon^{e}$ stand, respectively, for the finite element strain, stress, exact strain, and exact stress. 
This error cannot be computed exactly, since the fields to integrate are singular. We could have used our integration rule to measure the error. Experiment proved that this is not necessary, and instead, we used the simple partition integration rule, with order 5 (seven points per cell), to evaluate the error. The error is reported in Fig. 16 as a function of the total number of integration points used to compute the stiffness matrix. As can be seen, while with the partition integration the error converges very slowly and with oscillations, the new scheme displays an exponential convergence rate and reaches very small relative energy error. The best partition integration (order 19) gave an error of $7.2 \times 10^{-4}$ for a total of 89,644 integration points, while the new algorithm gave an error of $2.99 \times 10^{-5}$ for a total of 88,445 points, and the highest order used ( 20 points in the underlying one-dimensional Gauss-Legendre rule) reached an error of $1.13 \times 10^{-9}$ for 722,000 points.

\subsubsection{Adaptive Strategy}

On a reasonable scale problem like the one we just showed, one can ask about the penalty of a new approach in total computation time. Indeed, for the partition integration scheme, the integration points are known and are the same in the reference cell for all the integration cells. While in the case of our algorithm, for each cell, subcells must be constructed in the $(\rho, \tau)$ plane, and the integration point positions are the same for each subcell only in the $(s, t)$ plane. In the context of a classic finite element method, this would probably be an unacceptable price to pay. Indeed, all the shape functions in classic finite element method are defined on a reference element, and the evaluation of the shape function at each integration point can be done once and for all, in the reference element. Only the jacobian must be computed separately for each element. In the context of the X-FEM, the enrichment functions are defined as a function of the position of the point of evaluation in the global frame. There is no possibility to evaluate the shape function without knowing the coordinate of the node in real space. Whatever the method to construct the integration points, which can be viewed as a preprocessing step done once for a given mesh and given crack, the enrichment functions need to be computed at each integration point of each element. At equal numbers of integration points, the time to build the stiffness matrix is equal, whatever method is used to construct them. In our experiments, the time to construct the integration points was always so negligible compared to the rest of the solution process that we did not

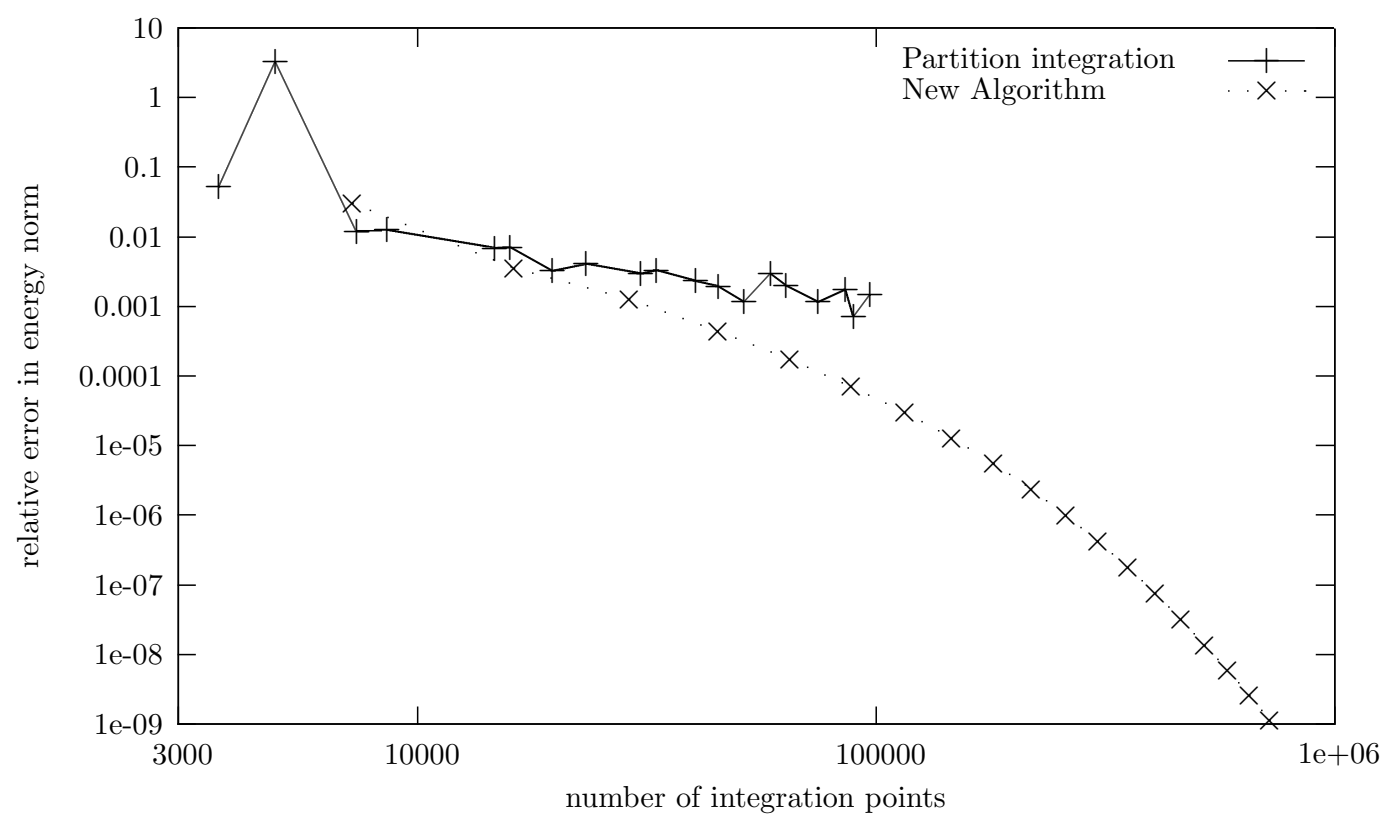

FIG. 16: Relative energy error norm, for a computation on a full mesh using enrichment everywhere, using partition integration and the new algorithm as a function of the total number of integration points. 
even bother to save them from one computation to another. They are computed when they are needed by the assembly process.

Nevertheless, if we could achieve a similar accuracy as the one just presented with fewer integration points, we would get faster assembling process. In order to achieve that, we will need some sort of adaptive strategy. Indeed, experiments showed that even with a small number of integration points, the new integration strategy gives very high accuracy in the local stiffness for most of the elements of the mesh. Only a few of them need a high number of points. Unsurprisingly, those elements are usually the one close to the crack tip. The direction is therefore clear. We could adaptively set the number of points of the underlying one-dimensional Gauss-Legendre rule from one cell to another if we had some sort of a priori error indicator for constructed rule over a cell. Our experiments have shown that the error in the evaluation of the area of a triangle using our integration scheme was strongly correlated to the error in the local stiffness matrix. This error, when all the points are mapped to the reference element, is very easy to evaluate: just sum all the weight of the integration points and compare to 0.5 , the exact area of the reference element. So, for each element, we construct all the subcells and then iteratively construct the integration points for an underlying onedimensional rule of increasing order until a threshold in the relative area error is reached. The experiment is reported in Fig. 17, where the relative energy error norm is plotted against the total number of points, for our integration algorithm with or without adaptivity, on the previous benchmark.

For the adaptive version, we set our parameter so that the minimum and maximum number of points of the onedimensional Gauss-Legendre rule are 3 and 49, respectively. For each point of the curve, we set a different value of the relative area error threshold, starting at $1.0 \times 10^{-2}$, and dividing by 10 for each new point until we reach our minimum threshold of $1.0 \times 10^{-13}$. As clearly shown, a huge gain in accuracy at equal number of integration points is achieved. The best reported adaptive result is reached for the smallest relative area error threshold of $1.0 \times 10^{-13}$. The energy error norm is then $3.476 \times 10^{-10}$ for a total of 161,271 points, compared to the 722,000 points obtained without adaptivity for an error still 3 times higher $\left(1.13 \times 10^{-9}\right)$.

The last version of our integration method is referred to as the singular integration in the rest of the text. The singular integration is parametrized by the minimum and maximum number of points in the underlying Gauss-Legendre rule (minpt and maxpt, respectively) and the area error threshold (ae).

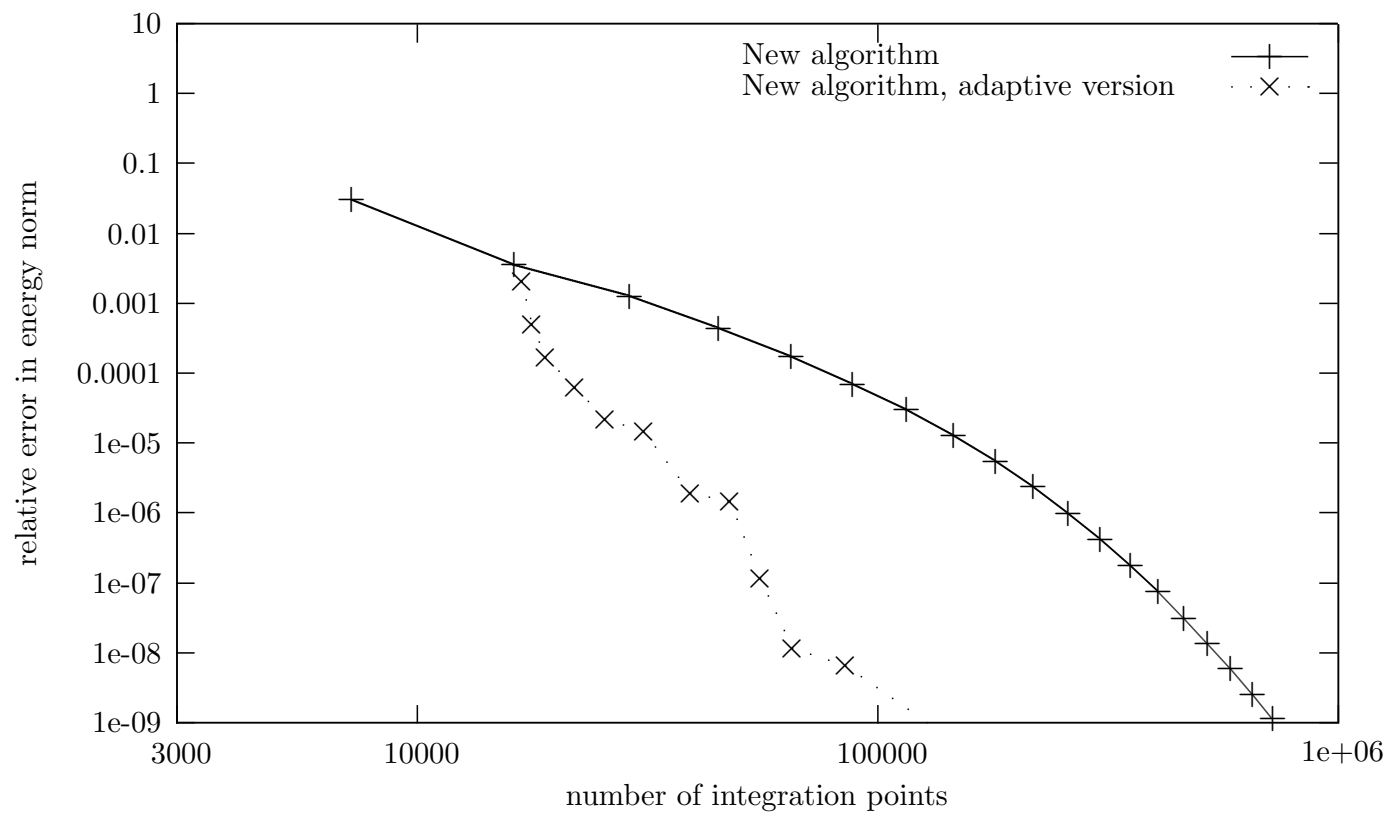

FIG. 17: Relative energy error norm, for a computation on a full mesh using enrichment everywhere, using the new algorithm, with or without adaptivity, as a function of the total number of integration points. 


\subsubsection{Comparison with a Scheme that Properly Treats Singular Cells Only}

Up to this point, we only compared our integration strategy with the quite crude partition integration. In the following we compare it with a more advanced scheme presented in Nagarajan and Mukherjee (1993) and Park et al. (2009), and apply it to our setting. We call this strategy polar mapping. In the polar mapping strategy, elements are split into integration cells in the same fashion as in partition integration. Weakly singular cells are integrated using one of the Gauss quadrature rules over the triangle, and singular cells are treated by using a the following "quasipolar" change of variable

$$
\xi=r \cos ^{2}(\theta) ; \quad \eta=r \sin ^{2}(\theta)
$$

where $r$ takes values in $[0: 1]$ and $\theta$ in $[0: \pi / 2]$ and again, $\xi$ and $\eta$ are the coordinates of a reference element, such as the singular node is mapped on $(0,0)$ in the $(\xi, \eta)$ plane. The integration rule is then constructed using a tensor product of the one-dimensional Gauss-Legendre rule. The polar mapping strategy therefore has two controlling parameters: the order $o$ of the integration rule in the weakly singular cell and the number npt of Gauss-Legendre points in each direction of the $(r, \theta)$ map. Figure 18 compares the results between the polar mapping strategy, the partition integration, and the adaptive singular integration, on the same test case. For the polar strategy, $o$ is increased from 6 to 19, while $n p t$ is set to 20. One can see that the polar strategy is of course better than the partition integration. The error is much less for an equivalent number of integration points: 97,012 points for an error of 0.0014 is the partition integration case, and 91,606 points for an error of $6.57 \times 10^{-5}$ in the polar mapping case. The adaptive singular integration with 86,704 points gives an error of only $6.52 \times 10^{-9}, 4$ orders of magnitude smaller than the polar mapping strategy. An error of $1.42 \times 10^{-5}$, slightly better than the best polar mapping, is obtained with 31,025 points, less than one third the number of points needed with the polar mapping. From the curves of the convergence of the polar mapping error, one can see that the error is not necessarily smaller when one increases the number of points. It does not seem to be the case with the adaptive singular mapping.

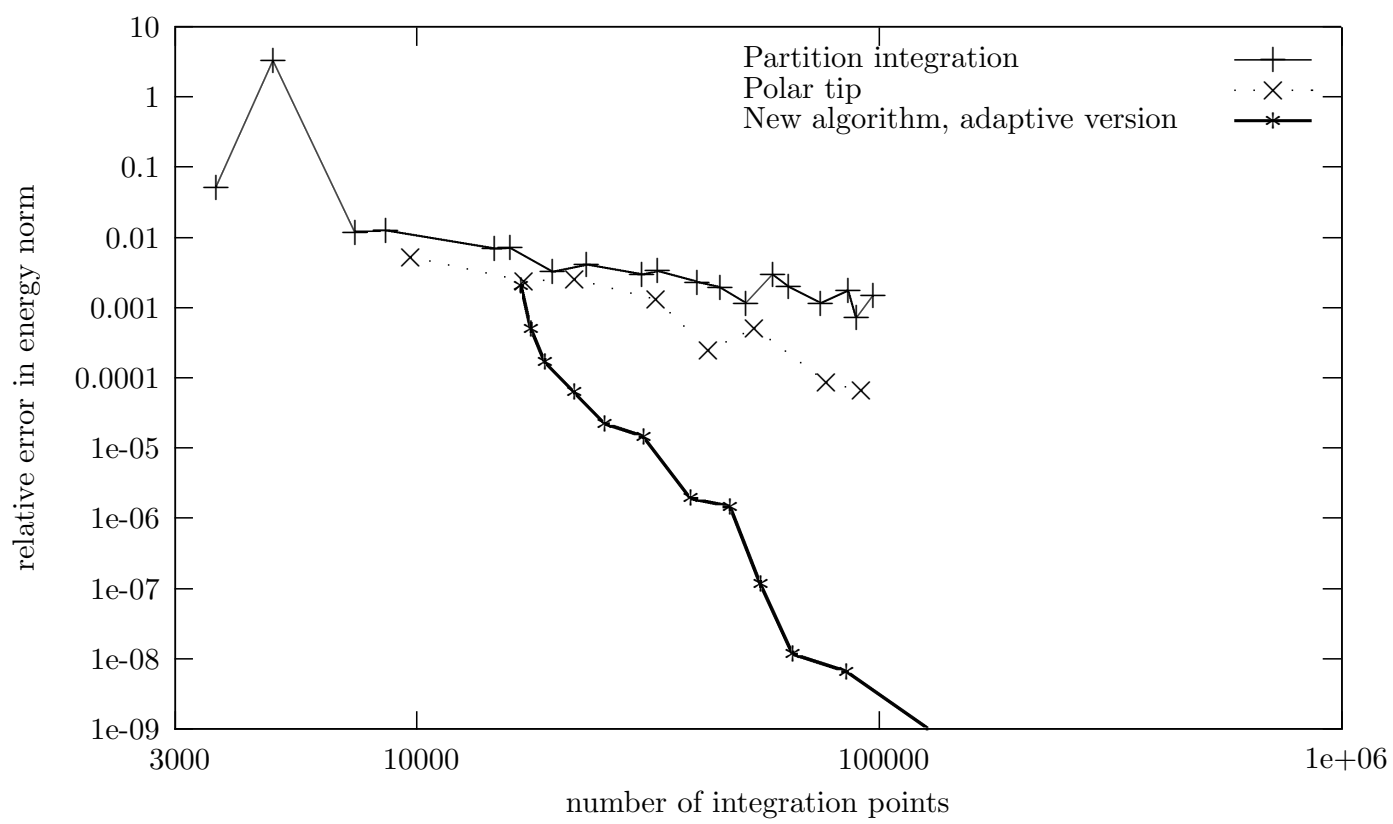

FIG. 18: Relative energy error norm, for a computation on a full mesh using enrichment everywhere, using the partition integration, the polar mapping, and the adaptive version of the new algorithm. 


\subsubsection{Robustness of the Scheme Compared to Polar Mapping}

In order to measure the robustness of the integration rule with respect to the relative position of the crack tip within an element, we next perform the following experiment. We take the same setting as in the previous experiment, we select the element where the crack tip is, and we do the same computation for different positions of the crack tip inside the element. More precisely, we will gradually move the crack tip $\mathbf{x}_{t}$ from the center of gravity $\mathbf{x}_{g}$ of the element to the closest point $\mathbf{x}_{c}$ to $\mathbf{x}_{g}$ on the boundary of the element. This experiment is parametrized by $s \in[0,1]$, such as $\mathbf{x}_{t}=(1-s) \mathbf{x}_{c}+s \mathbf{x}_{g}$. In Fig. 19, left side, the previous parametrization is depicted. We performed the same experiment as before, for different values of $s$, and measure the error in the energy norm using the polar mapping strategy or the singular integration. For this experiment, we took the best polar strategy used in the previous computation $(o=19$, $n p t=20)$ and the adaptive singular integration scheme that gave the same order of magnitude in the error ( $\operatorname{minpt}=3$, $a e=1.0 \times 10^{-7}$ ). The results of this experiment are depicted on the right of Fig. 19. In the case of the polar integration strategy, the magnitude of the error varies from a minimum of $1.2 \times 10^{-7}$ to a maximum of $7.8 \times 10^{-4}$, almost 4 orders of magnitude. This contrasts with the singular integration strategy, which returns an error varying only from $1.09 \times 10^{-5}$ to $1.41 \times 10^{-5}$. This contrast can easily be explained. When the crack tip moves closer and closer to the boundary of an element, the integration cells of the element on the other side of the boundary are more and more close to the crack tip, and therefore closer and closer to singular. With the polar mapping strategy, those cells are integrated with the classical integration rule that gives worse and worse results, while with the singular integration strategy, the singularity is taken into account. We think that this is an important fact, never reported before in the X-FEM literature. If close to singular cells are not taken into account properly in the integration scheme, the error in the stiffness matrix is very much dependent on the mesh (more precisely, the relative position of the crack tip inside an element). Treating properly the singular cell only is not enough to insure the robustness of the stiffness matrix computation. This issue might be crucial in particular for crack propagation problems where one as no control of the relative position of the crack tip of the element.

This concludes, for now, our work on the integration scheme for enriched elements. Starting from an efficient integration rule for singular cells (the parabolic mapping), we extended it in this contribution to weakly singular cells. This new integration scheme is efficient and accurate on any element, whatever its position relative to the crack tip. We have shown in the end of this section that the scheme, at equal numbers of integration points performs better than the partitioning strategy and also gives better results than the polar mapping.
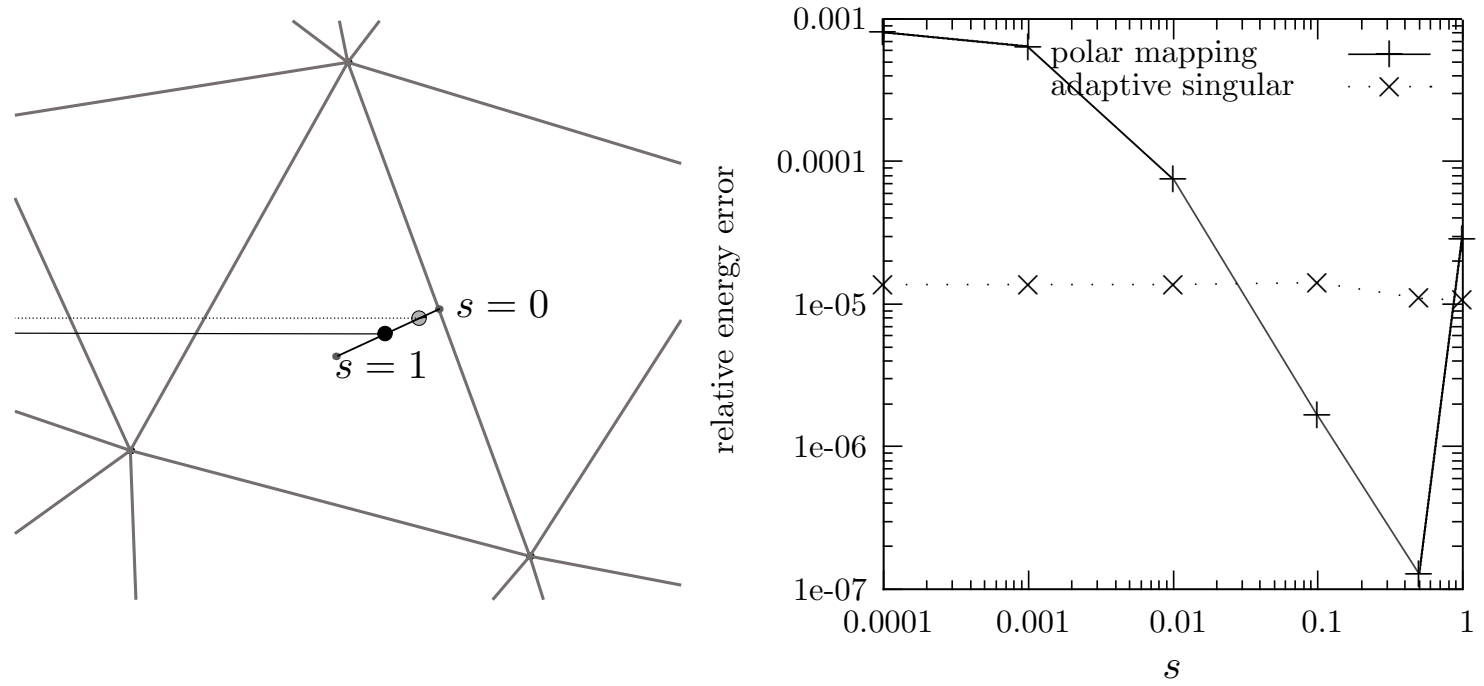

FIG. 19: Variation of the energy error with respect to the position of the crack tip. Left: parametrization of the crack tip position as a function of $s$. Right: error for both the polar mapping strategy and the adaptive singular integration. 


\section{NUMERICAL BENCHMARK AND CONVERGENCE TEST}

\subsection{Straight Crack}

In this section we propose to compare the two versions of the X-FEM methods. The analysis is focused on convergence rate and condition number. The test problem to solve is the two-dimensional problem of a planar crack in an infinite two-dimensional domain. The material is linear elastic and isotropic, and the computations are done in plane strain state. The analytical solution is known and is the Irwin solution. To solve this problem numerically, we work with a square domain, where the tensions of the analytical solution are applied on the boundary.

To track the convergence, we will always use the same series of meshes with increasing numbers of elements. The mesh are all unstructured meshes with as uniform as possible size field, obtained using the Gmsh package. The controlling parameter for the mesh size is $n$, the number of equal subdivisions along one edge of the square. The measure $h$ of the mesh size that we used for convergence analysis is the length of the square divided by $n$. Table 3 gives some characteristic measures for the series of mesh we used.

For all the presented results, the radius of geometrical enrichment for the tip enrichment function $R$ is set to 0.4. Elements which are not enriched by tip enrichment are integrated exactly using an integration rule on the triangular cell obtained by the splitting algorithm with an integration rule of order $2(p-1)$, where $p$ is the polynomial order of the shape function.

\subsubsection{Order One Shape Functions}

First we tested our series of mesh using the classical scalar tip enrichment function and used a linear shape function both for the classical field and the partition of unity. Due to the bad conditioning of the stiffness matrix, it was not possible to solve for mesh 7 in this case. In order to be able to solve up to mesh 6 , we had to use a minimal order of 5 for the partition integration in the enriched element. Analysis of the error is reported in Fig. 20 where the energy error is plotted as a function of the size of the element for each of the three integration schemes. In order to compare them properly, we set the parameters of each scheme so that we get approximately the same total of integration points for the finer mesh (around 200,000 points). The error is then mostly the same for each scheme, and the convergence rate is close to optimal.

Nevertheless, we looked more closely at the results. For instance, we compared, for different parameters of each integration scheme, the total number of integration points needed to reach a given precision. For a given mesh, we first compute a reference energy error, the energy error when using the best integration scheme that we have (typically the singular adaptive with ae set to $10^{-15}$ ). We then find for each scheme the set of parameters that return an energy error which is less than $1 \%$ larger than the reference energy error for the minimum number of integration points. This relative difference in energy error is clearly related to the effect of the integration error on the energy error. In the following we call it $\operatorname{err}_{i}$. The results on mesh 6 are reported in Table 4 . To reach the requested level of precision, the partition integration needed more than 500,000 points, while both polar and adaptive integration needed only around 200,000 points. These numbers show a slight edge for the adaptive singular scheme.

TABLE 3: Description of the series of meshes used for the convergence analysis

\begin{tabular}{|c|c|c|c|c|c|}
\hline Mesh number & $n$ & $h$ & Nodes & Edges & Elements \\
\hline 1 & 5 & 0.4 & 45 & 112 & 68 \\
2 & 10 & 0.2 & 145 & 392 & 248 \\
3 & 20 & 0.1 & 560 & 1597 & 1038 \\
4 & 40 & 0.05 & 2215 & 6482 & 4268 \\
5 & 80 & 0.025 & 8592 & 25453 & 16862 \\
6 & 160 & 0.0125 & 34172 & 101873 & 67702 \\
7 & 320 & 0.00625 & 135706 & 405835 & 270130 \\
\hline
\end{tabular}




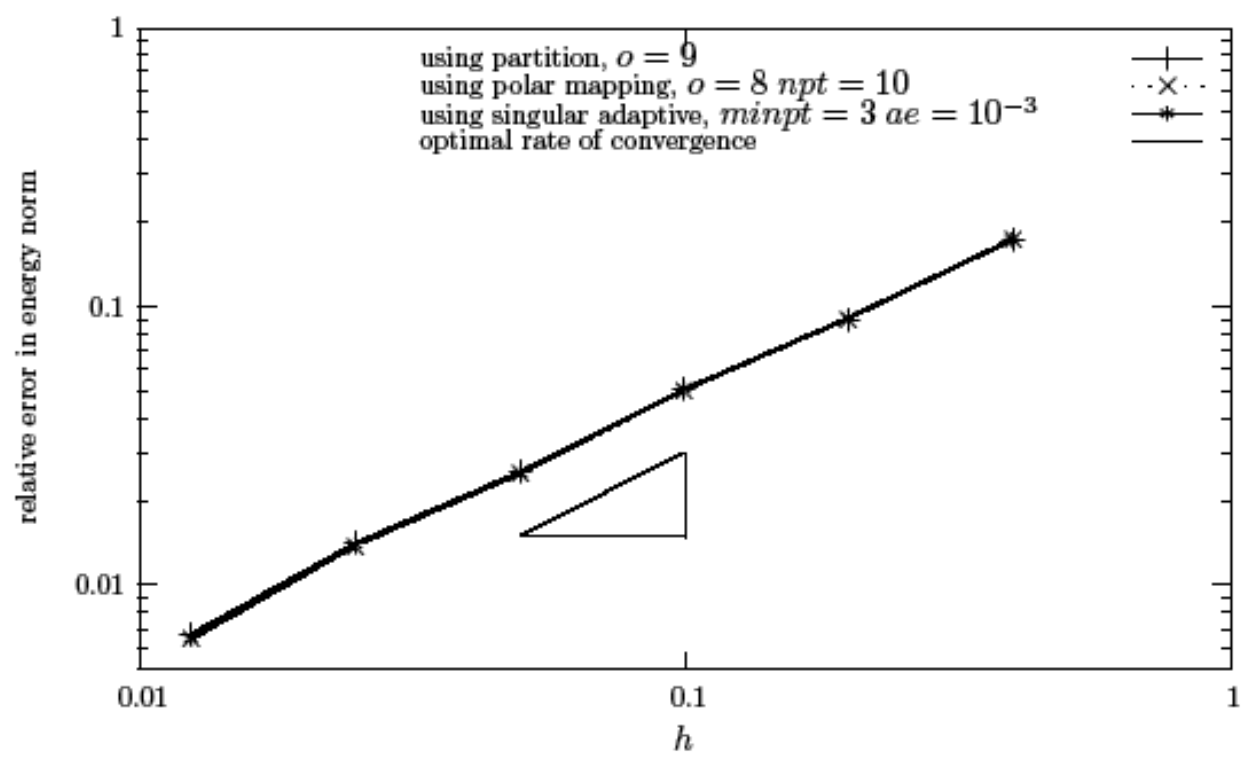

FIG. 20: Convergence analysis, linear shape functions, tip enrichment.

TABLE 4: Minimum number of points for each scheme and associated parameter to reach a precision better than $1 \%$ in the energy error

\begin{tabular}{|c|c|c|c|}
\hline Scheme name & Parameters & Number of points & $e r r_{i}$ \\
\hline Partition & $o=17$ & 513084 & $0.44 \%$ \\
Polar mapping & $o=8, n p t=10$ & 211380 & $0.79 \%$ \\
Adaptive singular & minpt $=3, a e=10^{-3}$ & 194072 & $0.05 \%$ \\
\hline
\end{tabular}

\subsubsection{Order 2 Shape Functions}

When moving to order 2, the situation changes drastically. We now set the order of the shape function to 2 , for both the classical field and the Heaviside enrichment. The tip enrichment is still multiplied by a partition of unity of order 1, since we found that it does not affect the convergence rate and that it maintains the conditioning to acceptable values. Again we computed the error as function of the mesh size with the three different schemes, setting the parameter so that we get the smallest error with approximately the same total of integration points for the finest mesh (around 100,000). Results are reported in Fig. 21. Partition integration always stays far from the optimal rate of 2. The Polar mapping strategy already really improves the situation, but the results are off for the final mesh: the interpolation error becomes of the same order as the integration error. The integration scheme is not precise enough in this case. The optimal convergence rate is only recovered with the adaptive singular rule, for this total of integration points. As in the first-order case, we have computed the best parameter set for each integration rule in order to get $e r_{i}$ less than $1 \%$ for mesh 5. Results are reported in Table 5. The partition integration could not reach the goal err $_{i}$; the best result was $\operatorname{err}_{i}=373 \%$ for the highest integration order. Both the polar mapping and the adaptive singular manage to reach this precision, the adaptive singular reaching the results with half the number of points needed by the polar mapping.

\subsubsection{Robustness of the Scheme}

To further prove the advantage of properly treating the weakly singular cell, we reproduced the experiment of moving the position of the crack tip inside an element gradually from the center of gravity to the boundary of the cell in our 


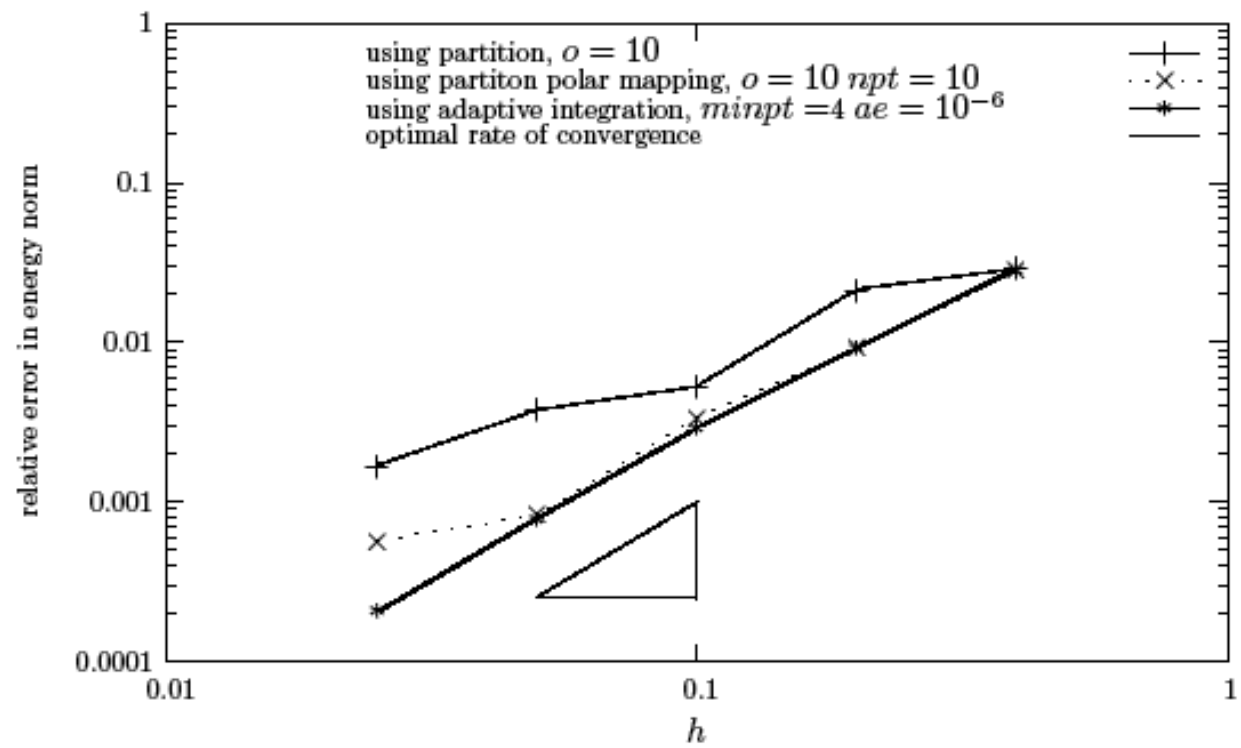

FIG. 21: Convergence analysis, quadratic shape function, tip enrichment.

TABLE 5: Minimum number of points for each scheme and associated parameter to reach a precision better than $1 \%$ in the energy error for order 2 shape functions

\begin{tabular}{|c|c|c|c|}
\hline Scheme name & Parameters & Number of points & $e r r_{i}$ \\
\hline Polar Mapping & $o=19, n p t=12$ & 235294 & $0.76 \%$ \\
Adaptive Singular & minpt $=4, a e=10^{-5}$ & 113887 & $0.69 \%$ \\
\hline
\end{tabular}

test case. Fig. 22 reports the energy error on mesh 5 for an order 2 shape function for the adaptive singular and the polar mapping integration rule as a function of the position of the crack tip inside the element, parametrized by $s$, which contained the crack tip in the previous numerical experiment. $s \in[0,1]$ is such as $\mathbf{x}_{t}=(1-s) \mathbf{x}_{c}+s \mathbf{x}_{g}$ as in Sec. 2.5.5. Whatever the position of the crack tip inside the element, the adaptive singular rule always gives the same error in the energy norm. In contrast, the polar mapping error is multiplied by more than 5 when the tip moves from $\mathbf{x}_{g}$ and approaches the element's boundary. The robustness issue that was already detected clearly has a damaging effect on the error for a real computation.

The singular integration rule permits integration, at low computational cost, with an error in the stiffness matrix that converges very fast to zero. But from our computations, it seems that this high degree of precision is only necessary when the discretization error is small enough for the error in the stiffness matrix to have an influence. For now, we can already draw the conclusion that the singular integration rule or any variant that can be proposed is a necessity when one wants to reach high accuracy and robustness.

\subsubsection{Vector Enrichment Function}

The same computations have been done using the vector enrichment function as presented in Sec. 1.4. The energy error is plotted as a function of the element size in Fig. 23. Again, using the singular integration rule, we reach an optimal order of convergence for both $p=1$ and $p=2$, with fewer degrees of freedom than with scalar enrichment. The total number of degrees of freedom, compared to the scalar enrichment case, is reported in Table 6. On our example, 


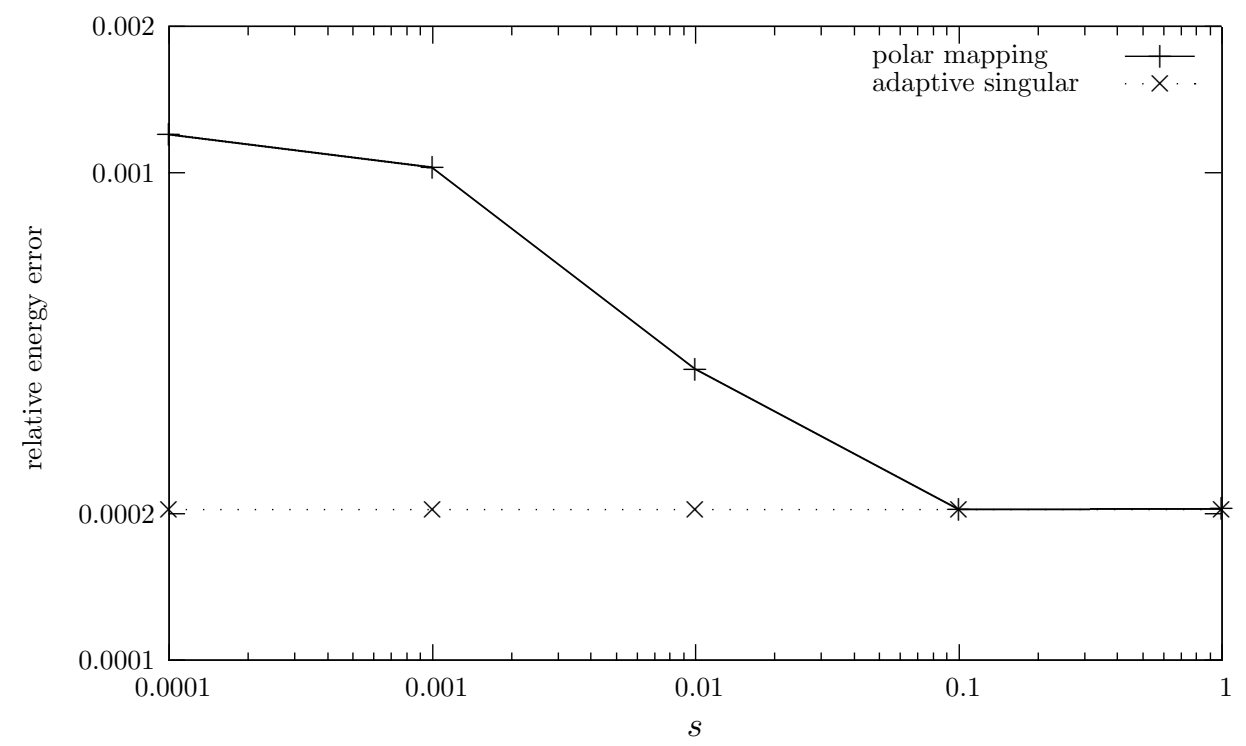

FIG. 22: Variation of the energy error with respect to the position of the crack tip for both the polar mapping strategy and the adaptive singular integration.

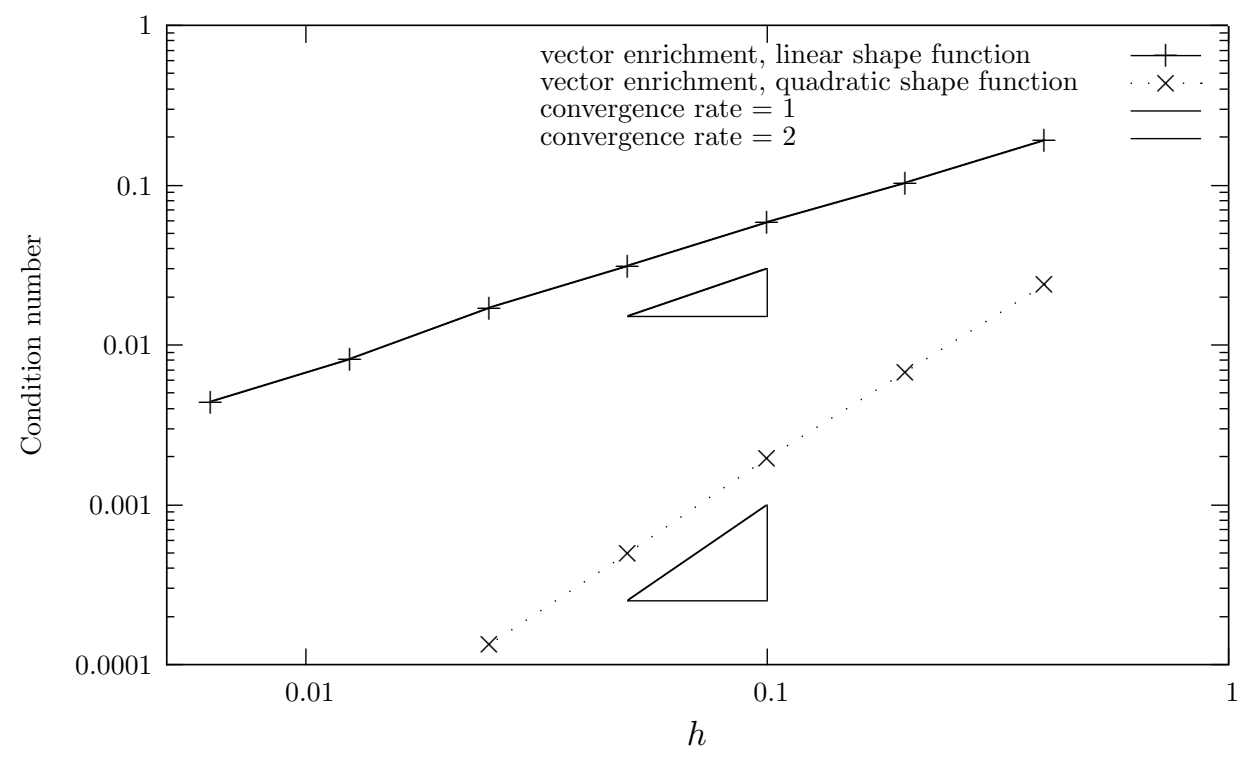

FIG. 23: Convergence analysis, linear and quadratic shape functions, vector enrichment.

the scalar enrichment generates about 30\% more degrees of freedom than the vector enrichment. More importantly, some cases that could not be solved in the scalar enrichment case worked perfectly with vector enrichment. This is due to the improved conditioning of the global stiffness matrix in the vector enrichment case. Figure 24 reports the condition number evolution as a function of the element size, for $p=1$, for both the scalar and vector tip enrichment strategies. On mesh 6, the condition number is as high as $2.93 \times 10^{15}$, for the scalar enrichment, and the problem could not be solved, as mentioned above, for mesh 7. On the other hand, using the vector enrichment function, the condition number was $1.24 \times 10^{8}$ and $2.46 \times 10^{9}$ for mesh 6 and 7 , respectively. 
TABLE 6: Number of degrees of freedom (ndof) per mesh as a function of the enrichment type

\begin{tabular}{|c|cccccc|}
\hline Mesh & $\mathbf{1}$ & $\mathbf{2}$ & $\mathbf{3}$ & $\mathbf{4}$ & $\mathbf{5}$ & $\mathbf{6}$ \\
\hline Ndof scalar enrichment & 139 & 463 & 1693 & 6649 & 25915 & 102991 \\
Ndof vector enrichment & 109 & 349 & 1297 & 5053 & 19507 & 77287 \\
\hline
\end{tabular}

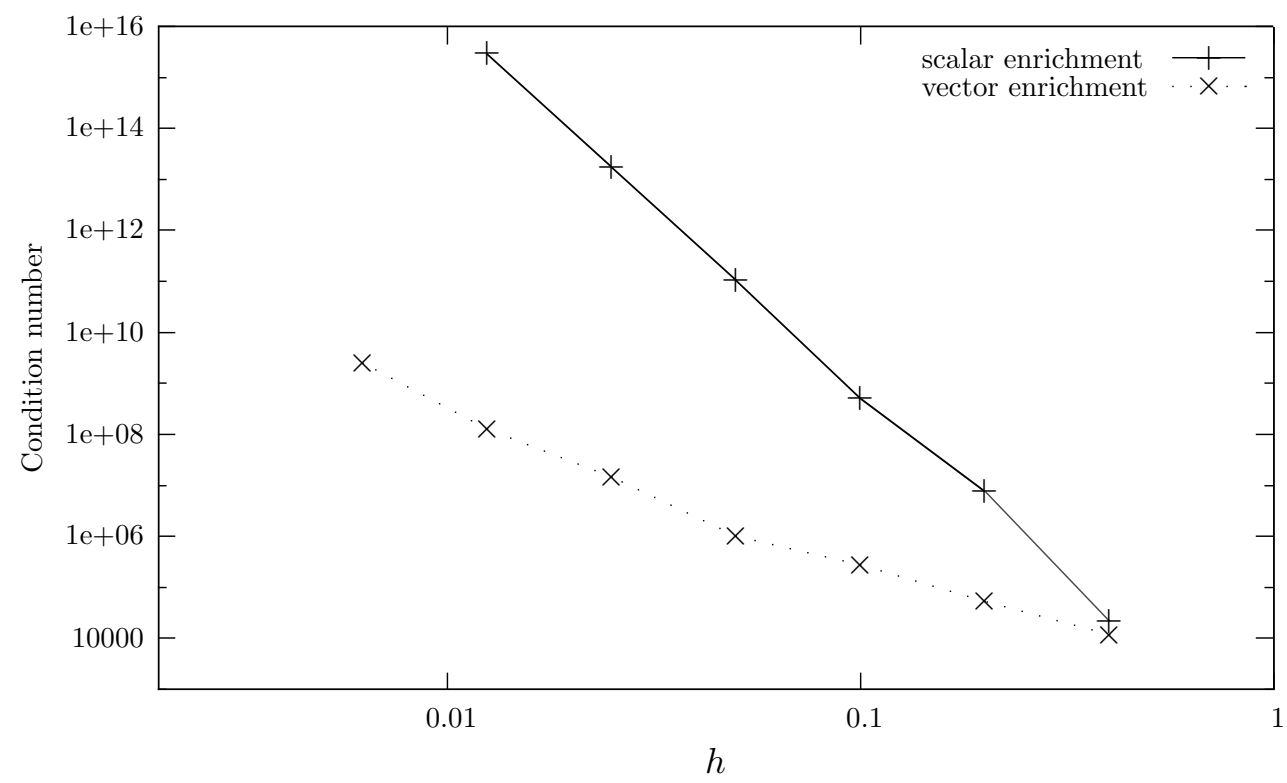

FIG. 24: Evolution of condition number of $\mathbf{K}$ with element size, using scalar of vector enrichment.

The conditioning problem only shows up when geometrical enrichment is used. When using topological enrichment, this problem usually does not occur, but the convergence rate is highly degraded and is not better that the one obtained with the ordinary finite element method. Topological enrichment is therefore a weak option when one wants to achieve high accuracy. Of course, the condition number problem was already noted before. Different solutions were proposed, like building an adhoc preconditioner as in Béchet et al. (2005) or by linking together values of the enrichment coefficients, for example, along disks centered around the crack tip in Laborde et al. (2005). The solution we proposed here, the vector enrichment function, is another answer to the problem, which has the advantage of avoiding to add any addition of another step to the method, and that reduces the number of degrees of freedom in the context of geometrical enrichment. The last remark is especially true in the three-dimensional case, where each time the mesh is refined, by a factor of 2, the number of nodes in the enrichment zone is multiplied by eight and the scalar enrichment asks for 12 additional degrees of freedom per enriched node, while vector enrichment only adds three per enriched node.

\subsection{Circular Arc Crack}

In this section, we present another important benchmark which again has an exact solution. We solve the problem of a circular arc crack in infinite media and compare with the analytical solution as found in Broberg (1999) and first explored in Muskhelishvili (1953) and Atluri et al. (1975). The solution in Broberg (1999) appears with mistakes in some of the coefficients. The corrected analytical solution can be found in Appendix 3.1. 
The definition of the problem can be seen in Fig. 25. The radius of the circle on which the crack lies is set to 1; the axis $x$ is the symmetry axis of the curved crack. The crack is bounded on an arc running from $-\theta$ to $\theta$, and loading at infinity is unidirectional stress, parametrized by its angle $\alpha$ and its magnitude $\sigma_{\infty}$.

This benchmark is important particularly for the vector enrichment function. Indeed, as was explained when we exposed this enrichment strategy, the enrichment functions are now dependent on the local crack direction. To simplify the implementation of the vector enrichment, we discretized these directions using a constant direction for each support of an enriched node, computed as the mean value of the gradient of the discretized level sets over this support. In the previous benchmark, the local crack axis was constant and the impact of its discretization could not influence the results. Here we wish to check if the optimal convergence rate is achieved for $p=1$. Convergence results are reported in Fig. 26 using the vector enrichment with a radius $R=0.15$.

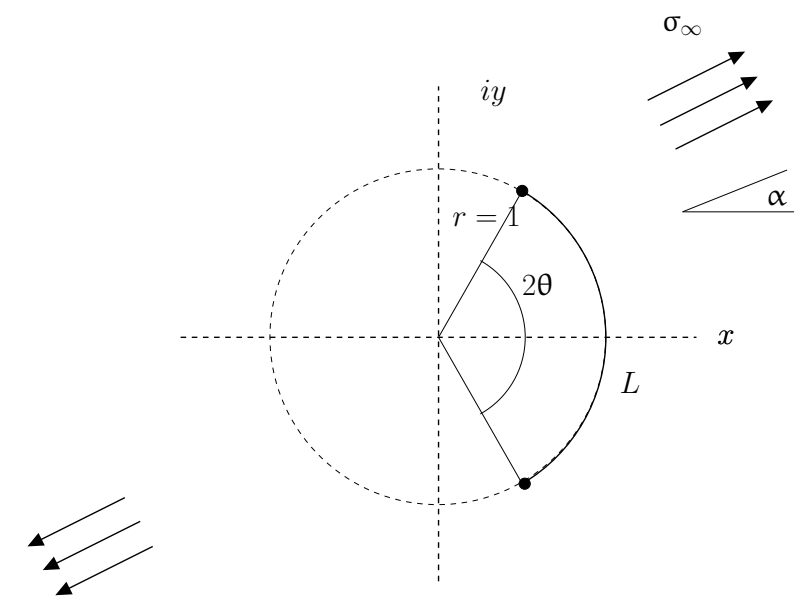

FIG. 25: Circular arc crack definition.

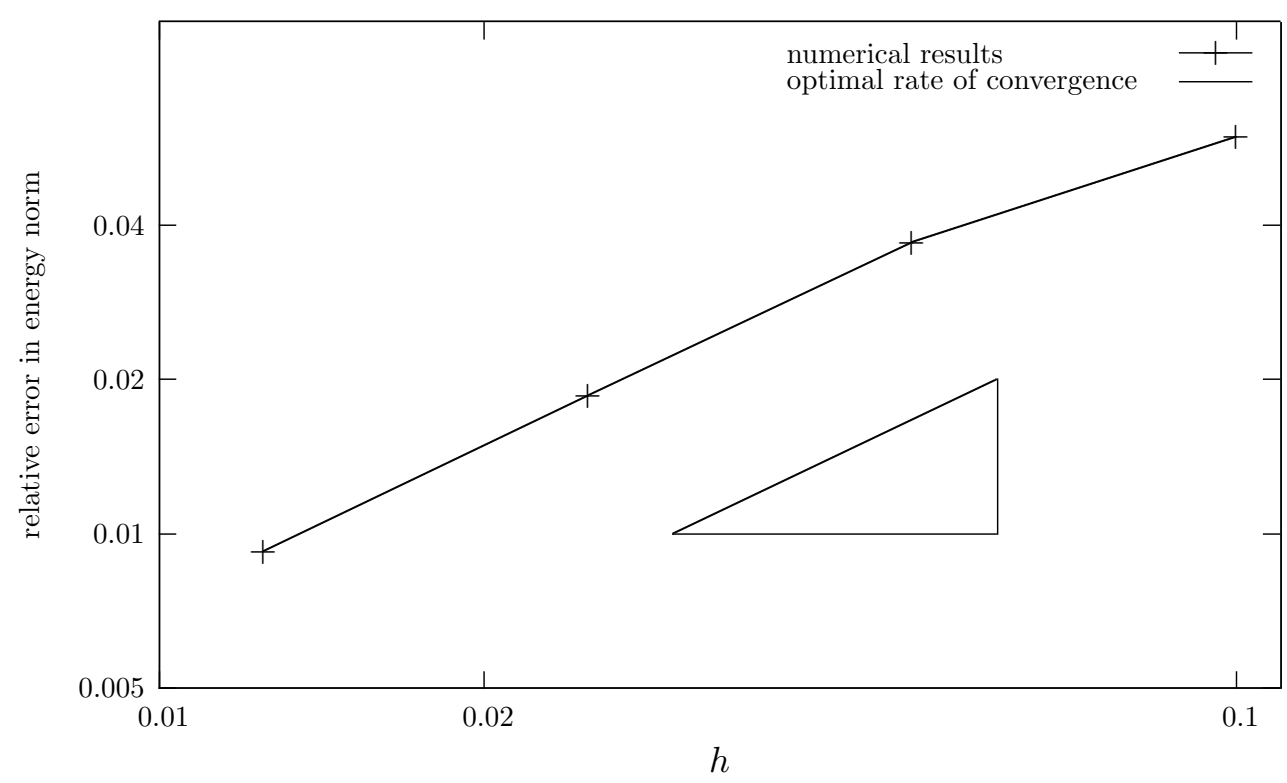

FIG. 26: Convergence results in energy norm for circular arc crack. 
The optimal rate of 1 for the energy norm is clearly obtained, which validates our discretization of the local crack axis. Typical images of the von Mises stress are displayed in Fig. 27 (left exact solution and right numerical solution).

In the present work, we remind that the level set is discretized linearly on the same mesh as the mesh to discretize the displacement field. There is no hope in this context to achieve optimal converge for higher order discretization of the displacement field since the error in the representation of the geometry would in this case destroy the convergence rate. In order to achieve optimal convergence rate in this cases, one needs to use a better representation of the geometry, as in Dréau et al. (2010) or Haasemann et al. (2011).

\subsection{Direct and Integral Extraction of Stress Intensity Factors}

The extraction of the stress intensity factor from a finite element computation is of major importance, particularly to predict failure, lifetime, or crack propagation in the structure. Many methods have been designed in the past and perhaps the most accurate are those based on domain integral methods or G-theta methods. The domain integral is an equivalent form to the well-known Rice contour integral (Rice, 1968). The theory behind the equivalent domain integral may be found in Moran and Shih (1987a,b). The use of domain integral in the finite element context started with the paper of Destuynder et al. (1983) for (2D) cracks and with Nikishkov and Atluri (1987) for 3D cracks. The concept of interaction domain integral to extract separately the stress intensity factors appeared in the work of Stern et al. (1976) for 2D cracks. The concept was then used to separate the stress intensity factors for 2D cracks (in Shih and Asaro, 1988, and 3D cracks Nakamura, 1991; Nakamura and Parks, 1989). The cracks considered were planar with straight edges. The axisymmetric cracks were then derived by Nahta and Moran (1993). Finally, general curved fronts were considered in Gosz et al. (1998) and Gosz and Moran (2002). It was since then applied to the X-FEM in Béchet et al. (2005), among others.

Our goal here is obviously not to compete with such methods, but the vector enrichment that we propose suggests a very efficient way to compute the stress intensity factors. We propose to interpolate linearly the value of the degrees of freedom associated with each of the vector enrichment functions, over the element that contains the crack tip at the position of the crack tip. The interpolation of the first degree of freedom should give an approximation of $K_{I}$ and the second one an approximation of $K_{I I}$. This is a postprocessing operation that can be done at no cost and which is very easy to implement once the vector enrichment functions are available in a given code. In the following, we call this method the direct evaluation method.

We have applied this strategy on the straight crack in infinite media in order to compare with the known analytical solution.
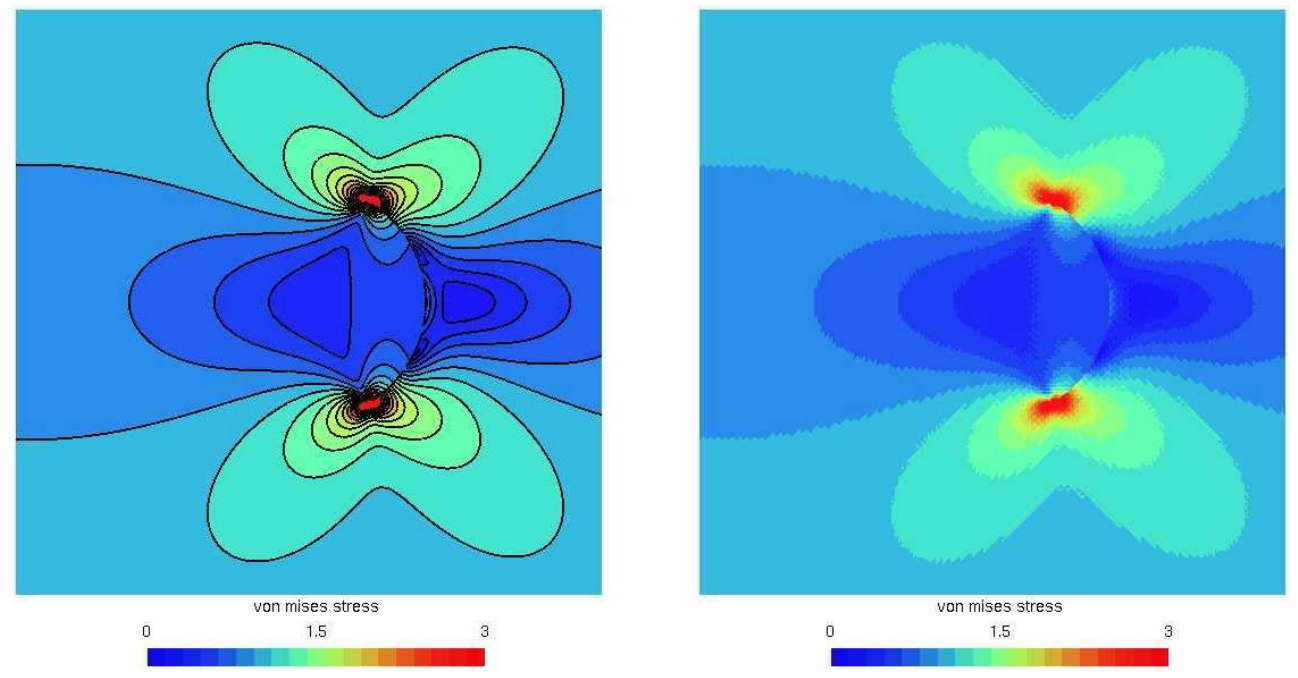

FIG. 27: Von Mises stress for the circular arc problem: (left) analytical solution and (right) exact solution. 
We also computed the stress intensity factors using a G-theta method on an integration domain defined geometrically, as it was shown that it is the most accurate strategy. The radius of the domain around the crack was set to the same value as the radius of the enrichment zone. Results using an order 1 shape function are plotted in Fig. 28, showing the error in the computed value of $K_{I}$ as a function of the element size. We recover, as expected, the optimal convergence rate of 2 for the G-theta method. The direct evaluation method also gives quite good results, considering its cost. The convergence rate is close to 1 .

Figure 29 reports the same results, but using order 2 shape functions. Again, an expected convergence rate of 4 is obtained for the G-theta method. The direct evaluation method gives also good results, but we probably need more refinement to correctly evaluate the convergence rate.

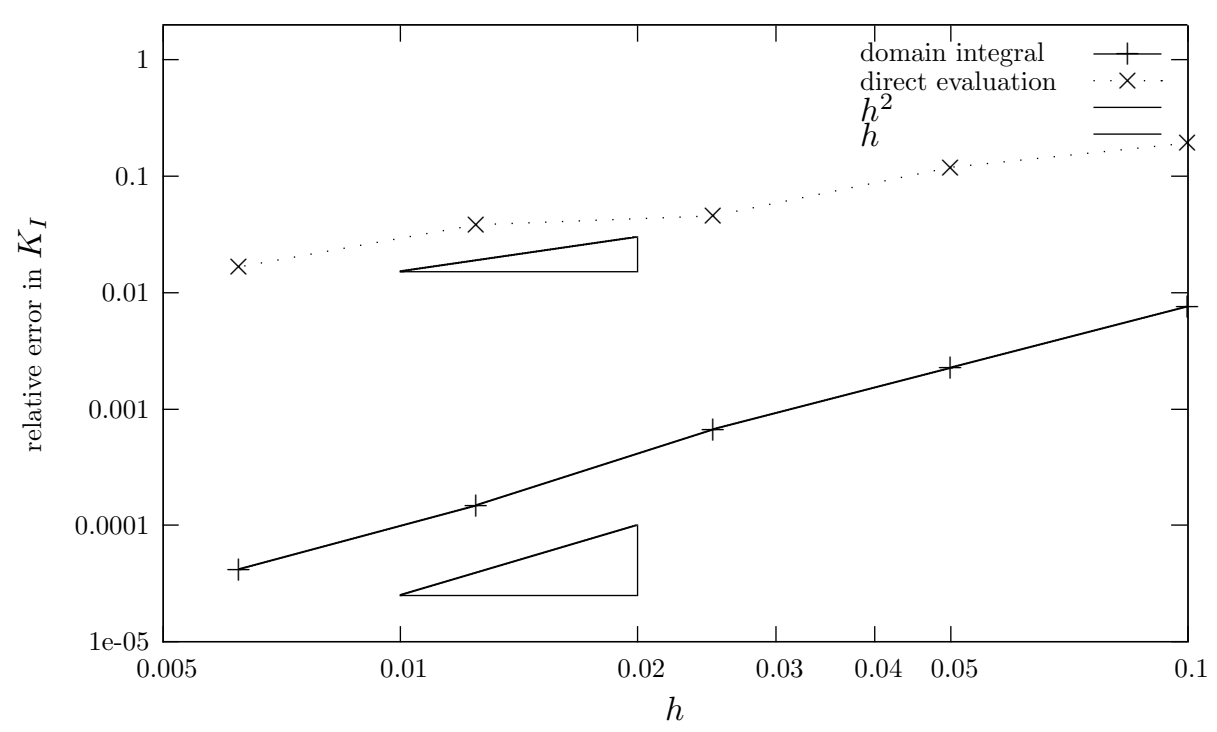

FIG. 28: Convergence results in the error in $K_{I}$, using first-order shape functions.

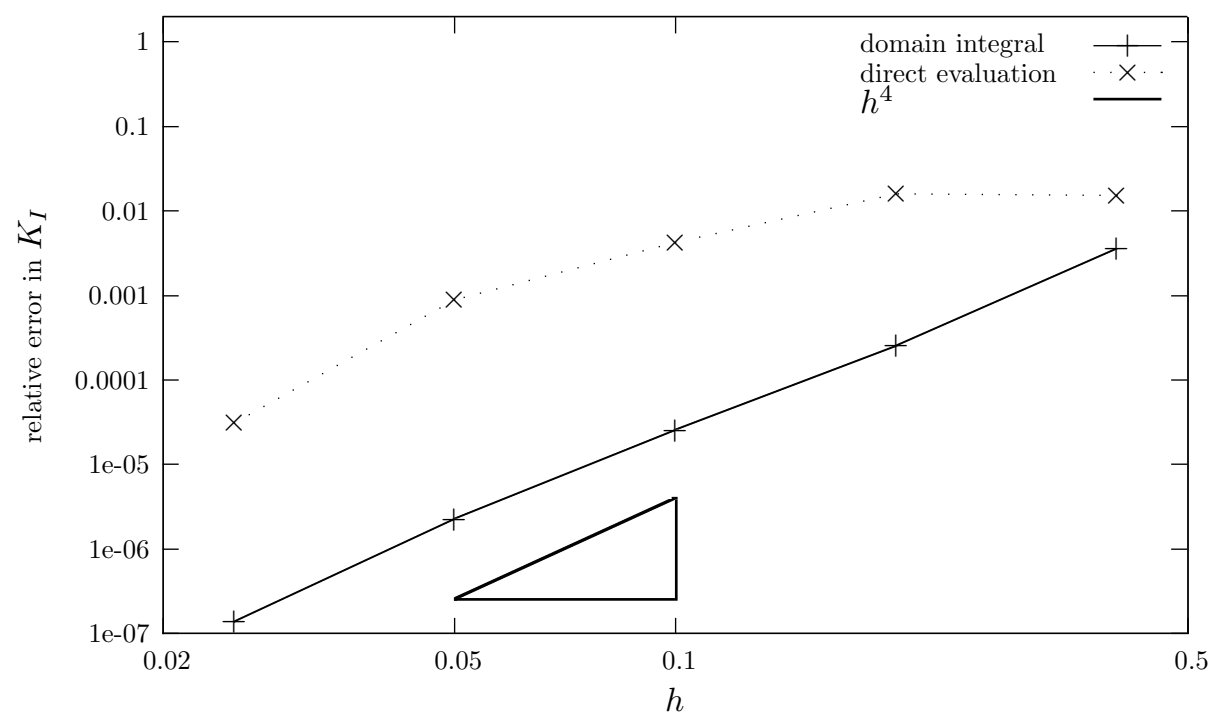

FIG. 29: Convergence results in the error in $K_{I}$ using second-order shape functions. 
Again, we do not advocate for the use of our direct evaluation method only: clearly the results of the G-theta method are much better. But having the possibility to quickly compute a rough evaluation of the stress intensity factor when a more precise one is available might prove useful nonetheless. Indeed, the difference between the two computed results can serve as an error indicator, giving an upper bound on the error committed by the G-theta method.

\section{CONCLUSIONS}

In this paper, we made two contributions to the X-FEM as applied to crack modelization in the context of linear elastic fracture mechanics.

- We advocate for the use of the vector crack tip enrichment function instead of the usual scalar crack tip enrichment function. Indeed, we have shown that for equivalent accuracy, it leads to a reduction of the number of degrees of freedom in the problem. It improves greatly the conditioning of the linear system to be solved and it offers a cheap extraction of the stress intensity factor.

- The design of a good integration rule to properly compute the local stiffness for tip-enriched elements containing, or close to, the singular tip was the object of much work. The paper presents a solution to this problem, including integration cells that do not contain the singularity. The only other proposed solution in the literature in these cases was the superposition method in Béchet et al. (2005). The method proposed in the current paper has the advantage of ensuring that all integration points are inside the elements and it is adaptive. It was shown that an accurate integration scheme for all enriched elements is important to obtain rapidly convergent values of the stiffness matrix, independently of the position of the crack tip inside an element. The effects of the quality of the integration rule are particularly important in the final error in case of a higher order discretization scheme.

We plan to pursue our work in the development of these special integration rules. The self-evident point is to extend our work for the three-dimensional case. We think that the hardest part is already done. Indeed, the singularity, even in full three dimensional case, is essentially two dimensional. In three dimensions, the problem of integrating properly over a tetrahedron can certainly be solved by cutting the tetrahedron into slices orthogonal to the crack front direction and solving the resulting two-dimensional integration problem. Alternatively, the tetrahedron could first be mapped to a prism aligned with the crack front direction, separating the integration into a series of two-dimensional problems. We already tried similar strategies and they are indeed promising, even if not yet sufficiently robust to be presented at the time the paper is being written.

We are also interested in providing integration rules adapted for other types of singularies. In particular, we have in mind to work on integration rules adapted to the integration of enrichment functions coming from the reentrant corner, where the singularity is of the form $r^{\alpha}$ with $\alpha$ dependent on the angle of the wedge.

\section{REFERENCES}

Atluri, S. N., Kobayashi, A. S., and Nakagaki, M., An assumed displacement hybrid finite element model for linear fracture mechanics, Int. J. Fracture, vol. 11, pp. 257-271, 1975.

Béchet, E., Minnebo, H., Moës, N., and Burgardt, B., Improved implementation and robustness study of the X-FEM method for stress analysis around cracks, Int. J. Numer. Methods Eng., vol. 64, pp. 1033-1056, 2005.

Belytschko, T. and Fries, T., The generalized/extended finite element method: An overview of the method and its applications, Int. J. Numer. Methods Eng., vol. 84, pp. 253-304, 2010.

Broberg, K. B., Cracks and Fractures, Academic Press, New York, 1999.

Demmel, J. W., Eisenstat, S. C., Gilbert, J. R., Li, X. S., and Liu, J. W. H., A supernodal approach to sparse partial pivoting, SIAM J. Matrix Anal. App., vol. 20, no. 3, pp. 720-755, 1999.

Destuynder, P., Djaoua, M., and Lescure, S., Some remarks on elastic fracture mechanics (quelques remarques sur la mécanique de la rupture élastique), J. Mécan. Théorique Appliquée, vol. 2, no. 1, pp. 113-135, 1983.

Dréau, K., Chevaugeon, N., and Moës, N., Studied X-FEM enrichment to handle material interfaces with higher order finite element, Comput. Methods. Appl. Mech. Eng., vol. 199, pp. 1922-1936, 2010. 
Duarte, C., Babuška, I., and Oden, J., Generalized finite element methods for three-dimensional structural mechanics problems, Comput. Struct., vol. 77, pp. 215-232, 2000.

Duarte, C. A., Hamzeh, O. N., Liszka, T. J., and Tworzydlo, W. W., A generalized finite element method for the simulation of three-dimensional dynamic crack propagation, Comput. Methods Appl. Mech. Engrg., vol. 190, pp. 2227-2262, 2001.

Duffy, M. G., Quadrature over a pyramid or cube of integrands with a singularity at a vertex, SIAM J. Numer. Anal., vol. 19, no. 6, pp. 1260-1262, 1982.

Galassi, M., GNU Scientific Library Reference Manual (3rd ed.), Network Theory Ltd., UK, 2009.

Geuzaine, C. and Remacle, J., Gmsh: A 3-D finite element mesh generator with built-in pre- and post-processing facilities, Int. J. Numer. Methods Eng., vol. 79, pp. 1309-1331, 2009.

Gosz, M., Dolbow, J., and Moran, B., Domain integral formulation for stress intensity factor computation along curved threedimensional interface cracks, Int. J. Solids Struct., vol. 35, pp. 1763-1783, 1998.

Gosz, M. and Moran, B., An interaction energy integral method for the computation of mixed-mode stress intensity factors along non-planar crack fronts in three dimensions, Eng. Fract. Mech., vol. 69, pp. 299-319, 2002.

Haasemann, G., Kästner, M., Prüger, S., and Ulbricht, V., Development of a quadratic finite element formulation based on the X-FEM and NURBS, Int. J. Numer. Methods Eng., vol. 86, pp. 598-617, 2011.

Irwin, G. R., Analysis of stress and strains near the end of a crack traversing a plate, ASME, J. Appl. Mech., vol. 24, pp. 361-364, 1957.

Laborde, P., Pommier, J., Renard, Y., and Salaun, M., High-order extended finite element method for cracked domains, Int. J. Numer. Methods Eng., vol. 64, pp. 354-381, 2005.

Legrain, G., Chevaugeon, N., and Dréau, K., High order X-FEM and levelsets for complex microstructures: Uncoupling geometry and approximation, Comput. Methods Appl. Mech. Eng., vol. 241, pp. 172-189, 2012.

Moës, N., Dolbow, J., and Belytschko, T., A finite element method for crack growth without remeshing, Int. J. Numer. Methods Eng., vol. 46, pp. 131-150, 1999.

Moës, N., Gravouil, A., and Belytschko, T., Non-planar 3D crack growth by the extended finite element and level sets. Part I: Mechanical model, Int. J. Numer. Methods Eng., vol. 53, pp. 2549-2568, 2002.

Moran, B. and Shih, C., Crack tip and associated domain integrals from momentum and energy balance, Eng. Fract. Mech., vol. 127, pp. 615-642, 1987a.

Moran, B. and Shih, C., A general treatment of crack tip contour integrals, Int. J. Fract., vol. 35, pp. 295-310, 1987b.

Mousavi, S. E. and Sukumar, N., Generalized duffy transformation for integrating vertex singularities, Comp. Meth. Appl. Mech. Eng., vol. 45, 2010.

Muskhelishvili, N. I., Some Basic Problems in the Mathematical Theory of Elasticity, Noordhoff, Groningen, Holland, 1953.

Nagarajan, A. and Mukherjee, S., A mapping method for numerical evaluation of two-dimensional integrals with $1 / r$ singularity, Comput. Mech., vol. 12, pp. 19-26, 1993.

Nahta, R. and Moran, B., Domain integrals for axisymmetric interface crack problems, Int. J. Solids Struct., vol. 30, no. 15, pp. 2027-2040, 1993.

Nakamura, T., Three-dimensional stress fields of elastic interface cracks, J. Appl. Mech., vol. 58, pp. 939-946, 1991.

Nakamura, T. and Parks, D., Antisymmetrical 3-d stress field near the crack front of a thin elastic plate, Int. J. Solids Struct., vol. 25, no. 12, pp. 1411-1426, 1989.

Nikishkov, G. and Atluri, S., Calculation of fracture mechanics parameters for an arbitrary three-dimensional, Int. J. Numer. Methods Eng., vol. 24, pp. 1801-1821, 1987.

Osher, S. and Fedkiw, R., Level Set Methods and Dynamic Implicit Surfaces, Springer Verlag, Berlin, 2002.

Park, K., Pereira, J. P., Duarte, C. A., and Paulino, G. H., Integration of singular enrichment functions in the generalized/extended finite element method for three-dimensional problems., Int. J. Numer. Methods Eng., vol. 78, no. 10, pp. 1220-1257, 2009.

Pereira, J. P., Duarte, C. A., Guoy, D., and Jiao, X., HP-generalized FEM and crack surface representation for non-planar 3D cracks, Int. J. Numer. Methods Eng., vol. 77, pp. 601-633, 2009.

Prabel, B., Combescure, A., Gravouil, A., and Marie, S., Level set X-FEM non-matching meshes: Application to dynamic crack propagation in elastic-plastic media, Int. J. Numer. Methods Eng., vol. 69, no. 8, pp. 1553-1569, 2007. 
Remacle, J., Chevaugeon, N., Marchandise, E., and Geuzaine, C., Efficient visualization of high-order finite elements, Int. J. Numer. Methods Eng., vol. 69, pp. 750-771, 2007.

Rice, J., A path independent integral and the approximate analysis of strain concentration by notches and cracks, J. Appl. Mech., vol. 35, pp. 379-386, 1968.

Sethian, J. A., Level Set Methods and Fast Marching Methods: Evolving Interfaces in Computational Geometry, Fluid Mechanics, Computer Vision, and Materials Science, Cambridge University Press, Cambridge, UK, 1999.

Shih, C. and Asaro, R., Elastic-plastic analysis of cracks on bimaterial interfaces: Part I — Small scale yielding, J. Appl. Mech., vol. 55, pp. 299-316, 1988.

Solin, P., Segeth, K., and Dolezel, I., Higher-Order Finite Element Methods. Studies in Advanced Mathematics, Chapman \& Hall/CRC, Boca Raton, FL, 2004.

Stern, M., Becker, E., and Dunham, R., A contour integral computation of mixed-mode stress intensity factors, Int. J. Fract., vol. 12, no. 3, pp. 359-368, 1976.

Sukumar, N., Chopp, D. L., Béchet, E., and Moës, N., Three-dimensional non-planar crack growth by a coupled extended finite element and fast marching method, Int. J. Numer. Methods Eng., vol. 76, no. 5, pp. 722-748, 2008.

Sukumar, N., Moës, N., Belytschko, T., and Moran, B., Extended finite element method for three-dimensional crack modelling, Int. J. Numer. Methods Eng., vol. 48, no. 11, pp. 1549-1570, 2000.

\section{APPENDIX: EXACT SOLUTION FOR CIRCULAR ARC CRACK}

The exact solution for a circular arc crack in infinite media can be' developed as follows. The origin is the center of the circle of radius 1 on which the crack lies. The $x$ axis is the axis of symmetry of the crack. The crack is defined between angle $-\theta$ and $+\theta$ from the $x$ axis. At infinity, the stress is supposed homogeneous and uniaxial, in the direction represented by angle $\alpha$, and its value is $\sigma_{\infty} . z$ is the complex representation of the coordinate of a point in the $(x, y)$ plane as defined above. $z=x+i y$ (see Fig. 25).

Let us first define some constant values:

$$
\begin{array}{ll}
b_{0}=-\frac{\sigma_{\infty}}{4} e^{2 i \alpha} & a_{0}=b_{0} \\
b_{1}=-\cos (\theta) b_{0} & a_{1}=1 \\
b_{2}=-\cos (\theta) b_{3} & a_{2}=\frac{\sigma_{\infty}}{4}-b_{3} \\
b_{3}=\frac{3 b_{0} \cos (\theta)^{2}-\sigma_{\infty}+2 b_{1} \cos (\theta)-b_{0}}{2[\cos (\theta)-3]} &
\end{array}
$$

Then the following complex potentials are defined:

$$
\begin{gathered}
f^{\prime}(z)=\frac{1}{G(z)} \sum_{n=0}^{3} b_{n} z^{n-2}+\sum_{n=0}^{2} a_{n} z^{n-2} \\
g(z)=(z)=\frac{1}{G(z)} \sum_{n=0}^{3} b_{n} z^{n-2}-\sum_{n=0}^{2} a_{n} z^{n-2}
\end{gathered}
$$

Where $G(z)$ is defined as:

$$
\text { if }\{(|z|<1) \quad \text { or } \quad[\operatorname{Re}(z)<\cos (\theta)]\} G(z)=\sqrt{z^{2}-2 z \cos (\theta)+1} \quad \text { else } \quad G(z)=-\sqrt{z^{2}-2 z \cos (\theta)+1}
$$


In the previous expression, $\sqrt{ }$. refers to the principal value of the complex square root $[\sqrt{(z)}=\sqrt{(}|z|) e^{i \arg (z) / 2}$ where $-\pi<\arg (z)<\leq p i]$

$$
\begin{gathered}
\Theta=2\left[f^{\prime}(z)+\bar{f}^{\prime}(z)\right] \\
\Phi=-2\left[z \bar{f}^{\prime \prime}(z)+\bar{g}^{\prime \prime}(z)\right] \\
\sigma_{x x}=\frac{1}{2} \operatorname{Re}(\Theta+\Phi) \\
\sigma_{y y}=\frac{1}{2} \operatorname{Im}(\Theta+\Phi) \\
\sigma_{x y}=\operatorname{Re}(\Theta)-\sigma_{x x}
\end{gathered}
$$

